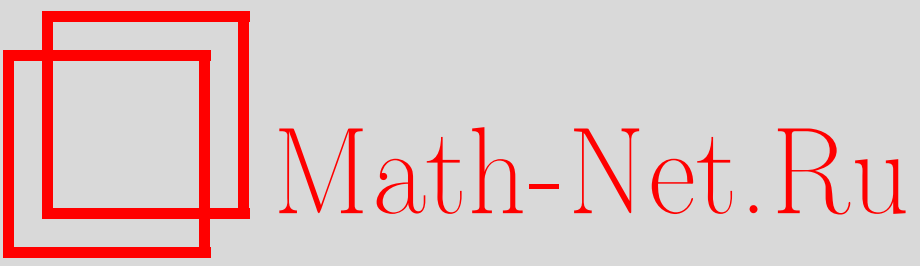

А. В. Забродин, Уравнение Хироты и анзац Бете, ТМФ, 1998, том 116, номер 1, 54-100

DOI: https://doi.org/10.4213/tmf889

Использование Общероссийского математического портала Math-Net.Ru подразумевает, что вы прочитали и согласны с пользовательским соглашением

http://www.mathnet.ru/rus/agreement

Параметры загрузки:

IP: 35.174 .16 .151

26 апреля 2023 г., 02:39:36 
ТЕОРЕТИЧЕСКАЯ

И МАТЕМАТИЧЕСКАЯ

ФИЗИКА

Том 116, № 1

июль, 1998

(C) 1998 г.

А. В. Забродин ${ }^{*}$

\section{УРАВНЕНИЕ ХИРОТЫ И АНЗАЦ БЕТЕ}

Приведен обзор недавних работ, посвященных анализу классических интегрируемых структур в квантовых интегрируемых системах, которые могут быть решены с помощью того или иного варианта анзаца Бете. Обсуждаются параллели между элементами теории тех и других. Некоторые ключевые объекты и понятия квантовой теории, ставшие стандартными в рамках квантового метода обратной задачи, отождествляются с типичными конструкциями теории классических солитонных уравнений. Функциональные соотношения между квантовыми трансфер-матрицами могут быть записаны в виде классического разностного уравнения Хироты, причем все основные результаты квантовой теории для спектра модели получаются путем решения этого классического уравнения. Обратно, решения этого последнего при наложении определенных граничных условий представляются с помощью типичных формул анзаца Бете. Знаменитое $T$ - $Q$-соотношение Бакстера и его обобщения появляются как одна из форм вспомогательной линейной задачи для уравнения Хироты.

\section{СОДЕРЖАНИЕ}

1. Введение . . . . . . . . . . . . . . . . . . . . . . . . . 55

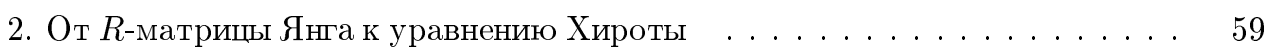

2.1. Процедура "размножения" $R$-матриц . . . . . . . . . . . . . . 59

2.2. Квантовые матрицы монодромии $\ldots \ldots \ldots \ldots \ldots \ldots \ldots \ldots \ldots$

2.3. Квантовые трансфер-матрицы . . . . . . . . . . . . 66

2.4. Функциональные соотношения и детерминантные формулы . . . . . 67

2.5. Билинейная форма правил слияния для прямоугольных диаграмм 68

2.6. Об эллиптическом и тригонометрическом случаях . . . . . . . . 69

3. Свойства решений уравнения Хироты, отвечающих квантовой задаче . 71

3.1. Граничные условия по $a$ и $s \quad \ldots \ldots \ldots \ldots \ldots \ldots \ldots \ldots \ldots \ldots$

3.2. Аналитические условия по $u$. . . . . . . . . . . . . . . . 73

3.3. Нормировка . . . . . . . . . . . . . . . . . . . 74

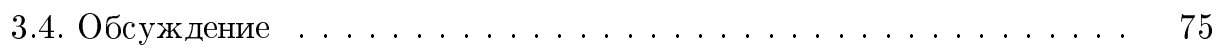

4. Иерархический анзац Бете как цепочка преобразований Беклунда . . . . . 75

* Объединенный институт химической физики, Москва, Россия; Институт теоретической и экспериментальной физики, Москва, Россия 
4.1. Представление нулевой кривизны и вспомогательные линейные за-

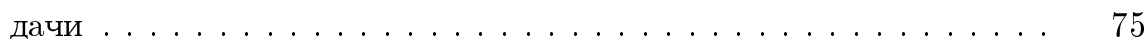

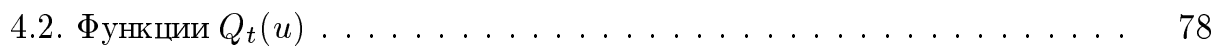

4.3. Уравнения Бете как динамическая система в дискретном времени . . 79

5 . Разностные уравнения для функций $Q_{t} \ldots \ldots \ldots \ldots \ldots \ldots$

5.1. Общее решение билинейных соотношений . . . . . . . . . . . . . . . 83

5.2. Обобшенные соотношения Бакстера . . . . . . . . . . . . . . . . . . . . 84

5.3. Формулы факторизации . . . . . . . . . . . . . . . . . 87

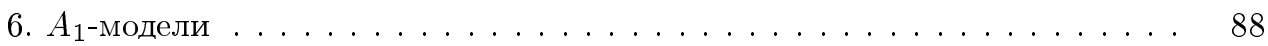

6.1. Общее решение дискретного уравнения Лиувилля . . . . . . . . . . . . 89

6.2. Различные формы соотношения Бакстера . . . . . . . . . . . . . . . . . 90

6.3. Высшие спины в квантовом пространстве . . . . . . . . . . . . . . . . . 91

6.4. Соотношение Бакстера как разностное уравнение для функции Бей-

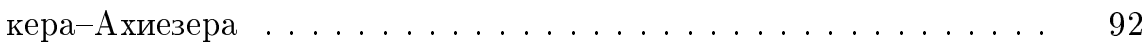

7. Заключение . . . . . . . . . . . . . . . . . . . . . . . . . . . . . . . . . . . . . . . .

Приложение . . . . . . . . . . . . . . . . . . . . 97

\section{1. ВВЕДЕНИЕ}

Для современного периода развития теории интегрируемых систем характерно взаимопроникновение методов, специфичных для классического и квантового случаев и ранее не пересекавшихся. Долгое время классические и квантовые интегрируемые модели изучались абсолютно различными методами, что не удивительно, поскольку в том и другом случаях нас обычно интересовали ответы на совершенно разные вопросы. Ошутимая польза от взаимодействия этих методов стала очевидной уже на самом раннем этапе развития квантового метода обратной задачи (КМОЗ) [1]. В результате анализа его классического предела было выявлено фундаментальное для современной гамильтоновой теории классических интегрируемых уравнений [2] понятие $r$-матрицы, универсальным образом задающей квадратичные алгебры скобок Пуассона.

В других отношениях теории классических и квантовых интегрируемых систем до сих пор развивались обособленно и выглядели как две разные области математической физики со своими понятиями, методами и традициями. Даже такой универсальный подход, как, например, метод обратной задачи, имеет совершенно разный смысл в квантовом и классическом контекстах, не говоря уже о более спещифических методах, вообше не имеющих аналогов в смежной области, таких как техника конечнозонного интегрирования (с классической стороны) или координатный анзац Бете (с квантовой стороны).

Постепенно становится ясно существование более глубоких связей между квантовыми и классическими интегрируемыми системами, чем просто тот факт, что последние получаются из первых процедурой, обратной процедуре квантования, т.е. в пределе $\hbar \rightarrow 0$. Оказывается, классические интегрируемые уравнения систематически появляются в квантовых интегрируемых задачах как точные соотношения и при $\hbar \neq 0$. Это 
явление было "экспериментально" обнаружено уже в большом количестве самых разных примеров. К исторически первым можно отнести полученные в [3] нелинейные уравнения, которым удовлетворяют корреляционные функции модели Изинга. В частности, было показано $[4,5]$, что парная корреляционная функция спинов в модели Изинга на квадратной решетке в точке самодуальности удовлетворяет разностному аналогу хорошо известного уравнения цепочки Тоды. Около десяти лет назад было обнаружено, что корреляционные функции интегрируемых моделей теории поля и во множестве других случаев удовлетворяют интегрируемым уравнениям классической теории (в обшем случае дифференциально-разностным) и список примеров этого типа был сушественно расширен $[6,7]$. К примерам другого типа относятся уравнения термодинамического анзаца Бете в модельно-независимой форме [8], а также функциональные соотношения для трансфер-матриц квантовых интегрируемых моделей $[9,10]$, которые могут быть представлены в виде классического разностного уравнения Хироты [11] (разностного аналога двумеризованной цепочки Тоды) или его "высших" аналогов. Другие аспекты подобных связей могут быть найдены в [12-16].

Есть основания ожидать, что интегрируемость сама по себе является глубоким и сильным свойством, способным придать новый смысл традиционному толкованию квантово-механического принципа соответствия. Постепенно накапливаются свидетельства в пользу того, что существует некая структура, обшая для классических и квантовых интегрируемых систем, разными проявлениями которой они являются. Образно говоря, если речь идет об интегрируемых системах, классический и квантовый случаи могут оказаться двумя сторонами одной медали. Подчеркнем, что указанная нетривиальная связь классических и квантовых задач наиболее отчетливо проявляется для ны $x$ классических уравнений, которые без всякого их дополнительного квантования уже содержатся в структуре квантовой теории.

Однако содержательный анализ этих интересных вопросов в такой обшей постановке - дело будущего. Возможно, создание такого объединенного формализма потребует адекватного описания динамических и корреляционных свойств квантовой системы, а не только спектральных. Здесь наша цель значительно скромнее. Мы, следуя работам [17-21], ограничимся выявлением классических интегрируемых структур в контексте спектральных свойств квантовых задач на конечных решетках в надежде, что это может служить хорошей отправной точкой для дальнейшего анализа.

Логическую структуру обзора можно проиллюстрировать с помошью диаграммы, приведенной на рис. 1.

"Подъем на вершину", т.е. попытка проследить весь путь от простейшей квантовой $R$-матришы к дискретным солитонным уравнениям, составляет содержание раздела 2. Большинство затронутых здесь вопросов за последние 15 лет неоднократно обсуждались в литературе. Мы пользуемся стандартными понятиями и соглашениями КМОЗ [22] без подробных комментариев. Стрелка, ведушая от $T$-матриц и гамильтонианов к уравнениям Бете, отвечает стандартной логике КМОЗ и здесь не обсуждается.

Дано краткое описание процедуры "размножения" $R$-матриц - построения более сложных из простейших путем "слияния" (fusion procedure) [23]. Самосогласованность 
Теория солитонов

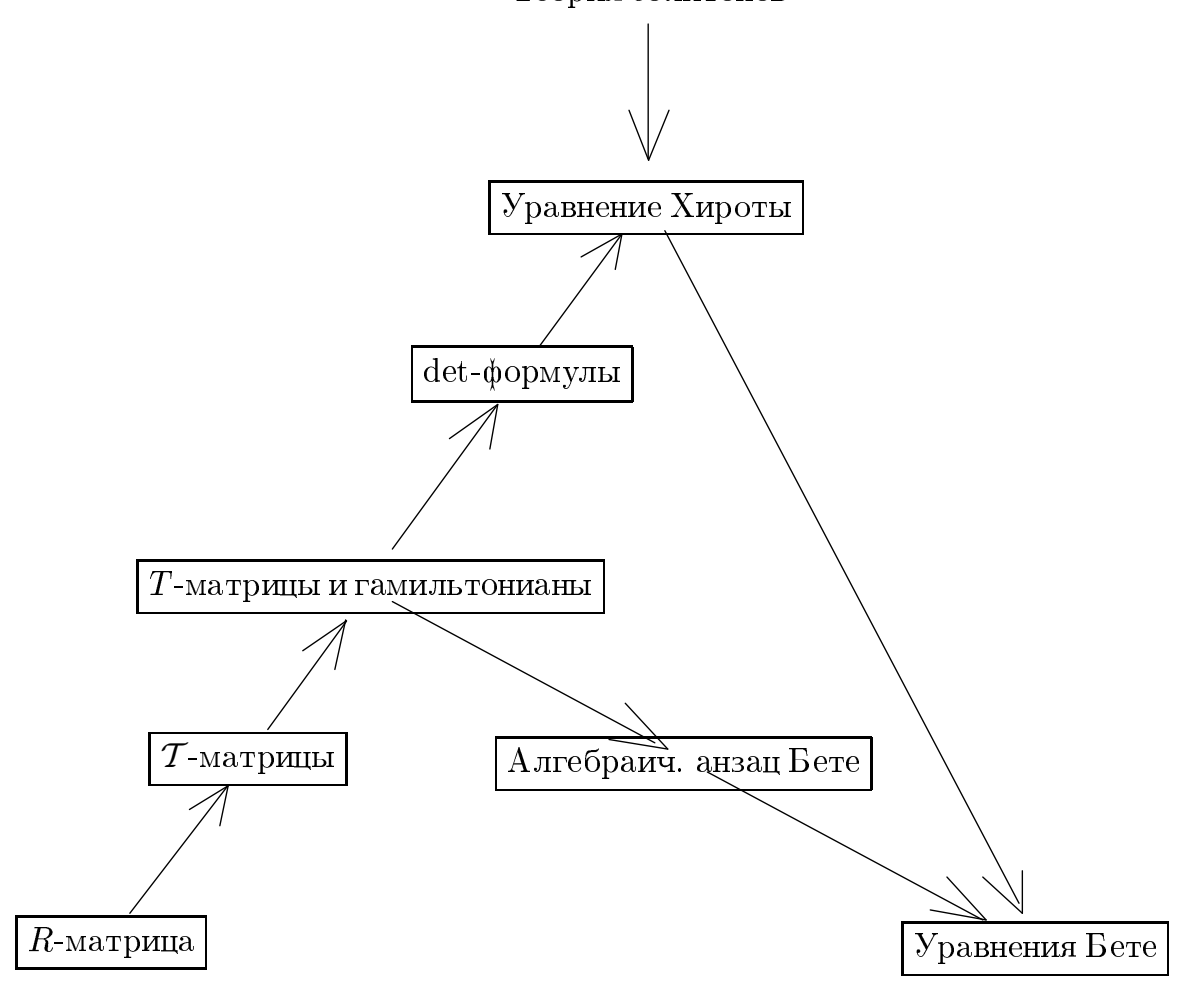

Рис. 1.

этой конструкции следует из уравнения Янга-Бакстера для $R$-матрицы. В результате получается семейство трансфер-матриц (производящих функций коммутирующих гамильтонианов), действующих в одном и том же квантовом пространстве. Они зависят от некоторых (спектральных) параметров и коммутируют меж ду собой при любых значениях этих параметров. (Чтобы прояснить идейную сторону дела, мы ограничились наиболее простым в техническом отношении случаем $R$-матриц с рациональной зависимостью от спектрального параметра.) Эти трансфер-матришы функционально зависимы и удовлетворяют ряду соотношений, которые иногда называют соотношениями слияния или правилами слияния. Они аналогичны тождествам для характеров линейных групп, но из-за наличия спектрального параметра имеют более сложную структуру. Будучи преобразованы к билинейной форме, соотношения слияния становятся универсальными (т.е. их вид перестает зависеть от конкретной модели).

Билинейная форма правил слияния - это вершина абстрактных конструкций в квантовой области, которые можно провести в рамках КМОЗ. Замечательно, что в этом месте они смыкаются с ключевыми объектами теории классических солитонных уравнений. 
Билинейные правила слияния имеют вид разностного уравнения Хироты

$$
T_{s}^{a}(u+1) T_{s}^{a}(u-1)-T_{s+1}^{a}(u) T_{s-1}^{a}(u)=T_{s}^{a+1}(u) T_{s}^{a-1}(u)
$$

для трехпараметрического семейства коммутирующих интегралов движения $T_{s}^{a}(u)$. Подчеркнем, что именно свойство квантовой интегрируемости (т.е. наличие многопараметрического семейства коммутативных операторов) позволяет свести операторные соотношения между ними к классическим уравнениям для их собственных значений. При этом различные квантовые состояния соответствуют различным решениям классического уравнения с определенными аналитическими свойствами и граничными условиями. Уравнение Хироты изначально возникло как универсальный дискретный аналог солитонных уравнений [24-28 $]^{1)}$. Этот факт свидетельствует о нетривиальной связи классических и квантовых интегрируемых систем, еще не понятой до конца.

Не пытаясь как-либо “объяснить” это явление, мы будем выяснять, какую практическую пользу можно из него извлечь. С третьего раздела начинается "спуск с вершины”. Соответственно точка зрения на уравнение Хироты меняется. Именно, оно трактуется здесь не как тождество, а как фундаментальное динамическое уравнение, которое (вместе с граничными условиями) позволяет полностью определить собственные значения гамильтонианов. При этом решения самого этого (классического!) уравнения предстают в виде формул анзаца Бете - факт сам по себе неожиданный и требуюший дальнейшего осмысления.

В разделе 3 проанализированы граничные и аналитические условия, выделяющие класс решений уравнения Хироты, отвечающий квантовым интегрируемым системам на конечных решетках. В разделе 4 показано, что иерархический анзац Бете можно понимать как цепочку последовательных преобразований Беклунда, "раздевающих" исходную задачу до дискретного уравнения Лиувилля и на последнем шаге до дискретного уравнения Д'Аламбера. Здесь же приведены аргументы в пользу того, что уравнения иерархического анзаца Бете естественно трактовать как уравнения движения некоторой динамической системы в дискретном времени, аналогичной системе Калоджеро-Мозера или Рейсенарса. В разделе 5 проведено окончательное отождествление указанных выше конструкций со стандартными элементами иерархического анзаца Бете, чем и завершается “спуск с вершины”. Доказательства некоторых утверждений вынесены в приложение.

По сравнению с работами [17-20] изложение в ряде мест упрошено. Вместе с тем добавлены некоторые доказательства и примеры, не вошедшие в эти статьи.

Раздел 6 посвящен подробному рассмотрению наиболее простого и знакомого примера моделей $A_{1}$-типа. В его последнем пункте предложена новая интерпретация $T$ - $Q$-соотношения Бакстера, вкладывающая его в контекст задач о "конечнозонном" интегрировании солитонных уравнений.

\footnotetext{
1) См. также наш обзор [29], в котором систематизированы основные сведения об уравнении Хироты.
} 


\section{2. ОТ R-МАТРИЦЫ ЯНГА К УРАВНЕНИЮ ХИРОТЫ}

\section{1. Процедура “размножения" $R$-матриц}

$R$-матрица. Фундаментальная рациональная $R$-матрица ( $R$-матрица Янга), действуюшая в $\mathbf{C}^{k} \times \mathbf{C}^{k}$, имеет вид

$$
R(u)=u I+2 \mathcal{P}
$$

где $I$ - единичная матрица, $\mathcal{P}$ - оператор перестановки: $\mathcal{P}(x \otimes y)=y \otimes x$, а $u$ - спектральный параметр. Записав оператор перестановки в терминах базисных $k \times k$-матриц $E_{i j}$ таких, что $\left(E_{i j}\right)_{m n}=\delta_{i m} \delta_{j n}$, имеем

$$
R(u)=u I+2 \sum_{i, j=1}^{k} E_{i j} \otimes E_{j i} .
$$

Эта $R$-матрица удовлетворяет уравнению Янга-Бакстера

$$
R_{12}\left(u_{1}-u_{2}\right) R_{13}\left(u_{1}-u_{3}\right) R_{23}\left(u_{2}-u_{3}\right)=R_{23}\left(u_{2}-u_{3}\right) R_{13}\left(u_{1}-u_{3}\right) R_{12}\left(u_{1}-u_{2}\right)
$$

и обладает свойством $G L(k)$-инвариантности. Обе части являются линейными операторами в $\mathbf{C}^{k} \times \mathbf{C}^{k} \times \mathbf{C}^{k}$. Мы используем следуюшие стандартные обозначения: $R_{12}(u)$ действует как $R(u)$ в тензорном произведении первых двух пространств и как единичная матрица в третьем пространстве, аналогично для $R_{13}, R_{23}$. Графически уравнение $(2.3)$ представлено на рис. 2.

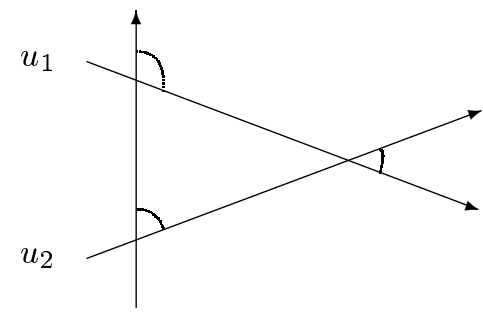

$u_{3}$

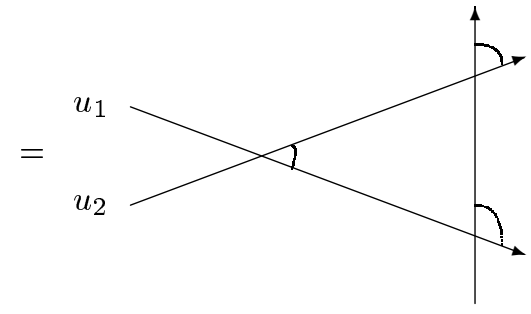

$u_{3}$

Рис. 2.

Пересечение двух линий изображает $R$-матрицу, зависяшую от разности спектральных параметров, ассоциированных с этими линиями. Свободные концы линий соответствуют свободным индексам матричного произведения (2.3), а по индексам, отвечающим концам внутренних отрезков, производится свертка. В графической интерпретации соотношение Янга-Бакстера означает, что каждую из трех линий можно переносить через точку пересечения двух других линий без изменения результата. 
Конструкция с лияния. Используя $R$-матрицу (2.1) как элементарный строительный блок, можно "размножать" решения уравнения Янга-Бакстера, строя из простых более сложные. Это достигается перемножением $R$-матриц с последующим проецированием на неприводимое представление группы $G L(k)$.

Пусть $\lambda=\left(\lambda_{1}, \lambda_{2}, \ldots, \lambda_{m}\right)$ - диаграмма Юнга из $n$ клеток с $m$ строками $\lambda_{1} \geqslant$ $\lambda_{2} \geqslant \cdots \geqslant \lambda_{m}, m \leqslant k$

$$
\sum_{i=1}^{m} \lambda_{i}=n, \quad P_{\lambda}: \bigotimes_{i=1}^{n} V_{i} \rightarrow V^{(\lambda)}
$$

- оператор проецирования на подпространство неприводимого представления $G L(k)$, соответствующего диаграмме $\lambda$. В клетку с координатами $(i, j)(i$-я строка и $j$-й столбец) впишем число

$$
s_{(i j)}=u-2(i-j)
$$

(например, см. рис. 3).

\begin{tabular}{|c|c|c|c|}
\hline$u$ & $u+2$ & $u+4$ & $u+6$ \\
\hline$u-2$ & $u$ & $u+2$ & \\
\hline$u-4$ & \multicolumn{3}{|c}{} \\
\cline { 1 - 2 } & \multicolumn{2}{|c}{} \\
\cline { 1 - 2 } & &
\end{tabular}

Рис. 3

Пронумеровав все клетки в "алфавитном" порядке (т.е. слева направо в первой строке, затем слева направо во второй строке и т.д.), получим упорядоченную последовательность чисел $s_{1}, s_{2}, \ldots, s_{n}$ (для приведенного выше примера это будет $s_{1}=u$, $\left.s_{2}=u+2, s_{3}=u+4, s_{4}=u+6, s_{5}=u-2, s_{6}=u, s_{7}=u+2, s_{8}=u-4\right)$.

По определению $R$-матрища в $V^{(\lambda)} \otimes \mathbf{C}^{k}$ имеет вид

$$
R^{(\lambda)}(u)=P_{\lambda} R_{n 0}\left(s_{n}\right) \otimes \cdots \otimes R_{20}\left(s_{2}\right) \otimes R_{10}\left(s_{1}\right) P_{\lambda}
$$

Фундаментальные $R$-матрицы $R_{i 0}\left(s_{i}\right)$ тензорно перемножаются в пространствах $V_{i}=$ $\mathbf{C}^{k}$, а их матричные элементы перемножаются в обшем для них пространстве $V_{0}=\mathbf{C}^{k}$ в указанном порядке. Графически это изображено на рис. 4.

На этом рисунке прямоугольник символизирует вставку проектора, а с жирной линией ассоциировано пространство неприводимого представления группы $G L(k)$, на которое он проецирует.

Пользуясь стандартной терминологией КМОЗ, мы будем называть пространство, ассоциированное с горизонтальными (вертикальными) линиями, вспомогательным (соответственно, квантовым). 


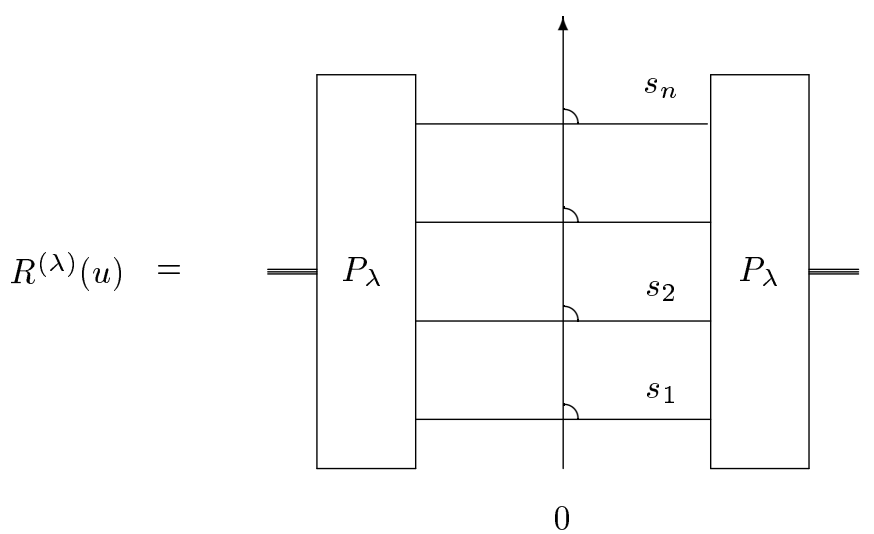

Рис. 4.

Теорема 2.1. $R^{(\lambda)}\left(\right.$ и) удовлетворяет уравнению Янга-Бакстера в $\mathcal{V}_{1} \otimes \mathcal{V}_{2} \otimes \mathcal{V}_{3}$ : $R_{12}^{(\lambda)}\left(u_{1}-u_{2}\right) R_{13}^{(\lambda)}\left(u_{1}-u_{3}\right) R_{23}\left(u_{2}-u_{3}\right)=R_{23}\left(u_{2}-u_{3}\right) R_{13}^{(\lambda)}\left(u_{1}-u_{3}\right) R_{12}^{(\lambda)}\left(u_{1}-u_{2}\right)$, әде $\mathcal{V}_{1}=V^{(\lambda)}, \mathcal{V}_{2}=\mathcal{V}_{3}=\mathbf{C}^{k}$

Идея доказательства состоит в том, чтобы представить проектор $P_{\lambda}$ в виде произведения фундаментальных $R$-матриц. Тогда можно перемешать все линии, пользуясь уравнением Янга-Бакстера (2.3). Однако аккуратное проведение этой идеи в общем случае довольно сложно с технической точки зрения. Ниже будут указаны основные шаги доказательства. Одновременно это поможет нам выяснить расположение нулей построенной $R$-матрицы, что важно в дальнейшем.

Ключевыми являются следуюшие два свойства $R$-матрицы (2.1):

а) существует такое значение $u$, при котором $R(u)$ пропоршиональна оператору перестановки

$$
R_{12}(0)=2 \mathcal{P}_{12},
$$

графически (рис. 5) это означает, что линии размыкаются;

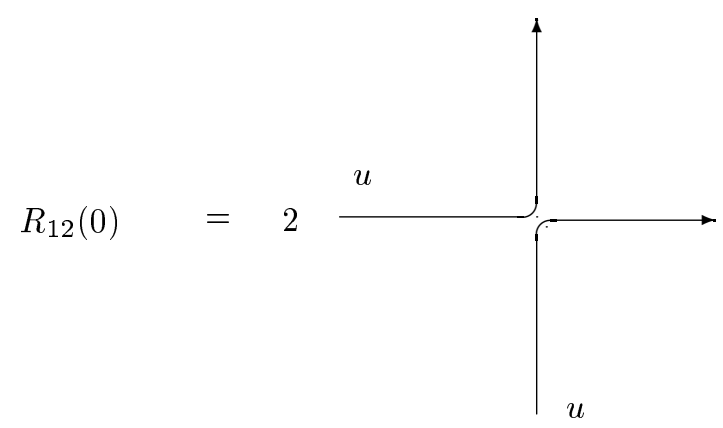

Рис. 5. 
б) $R$-матрица вырождается при $u= \pm 2$ :

$$
R_{12}( \pm 2)=4 P_{12}^{ \pm}
$$

где $P_{12}^{ \pm}$- проекторы на симметрические (антисимметрические) тензоры в $V_{1} \otimes V_{2}$.

Выше была определена упорядоченная последовательность спектральных параметров $s_{1}, s_{2}, \ldots, s_{n}$. Свойство "б" подсказывает, что в качестве проектора можно взять

$$
P_{\lambda}^{\text {пробный }} \propto \prod_{i<j}^{n} R_{i j}\left(s_{i}-s_{j}\right),
$$

где структура упорядоченного произведения считывается с графа полного пересечения $n$ линий, несущих спектральные параметры $s_{i}$. Порядок последовательных пересечений не важен в силу уравнения Янга-Бакстера (рис. $6, n=3$ ).

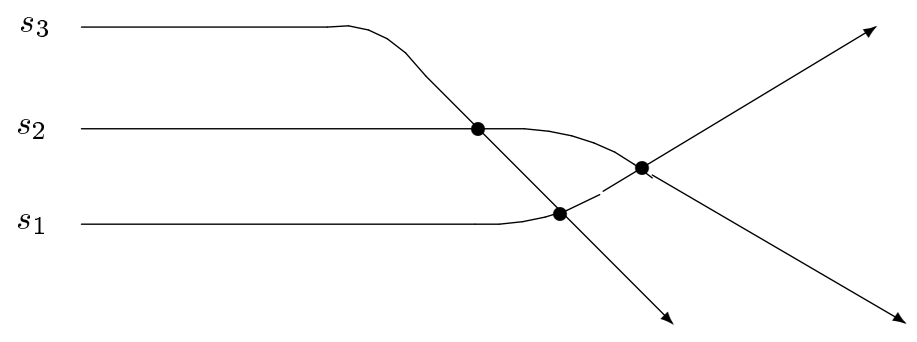

Рис. 6.

По индукции доказывается, что этот рецепт действительно работает для "крюков" $\lambda=\left(\lambda_{1}, 1,1, \ldots, 1\right)$ - диаграмм, любая диагональ которых содержит не более одной

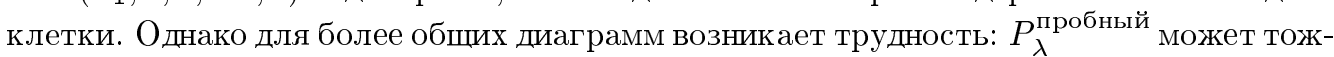
дественно обратиться в нуль. Например, если $\lambda=(2,2)$, мы имеем проектор, равньй нулю вследствие того, что дополнительные проекторы $P^{+}$и $P^{-}$оказываются умноженными друг на друга (рис. 7). Очевидно, что это происходит всякий раз, когда в диаграмме найдутся по крайней мере две строки $i \neq j$ такие, что $s_{i}=s_{j}$. Такие строки всегда найдутся, если в диаграмме имеется хотя бы одна диагональ (проведенная сверху вниз и справа налево), которая содержит более одной клетки. В этом случае требуется некоторая "регуляризация" наивного проектора (2.8), идея которой принадлежит Череднику [30].

Введем малый параметр $\epsilon$ и впишем в клетки $(i, j)$ диаграммы числа

$$
s_{(i j)}^{\epsilon}=u-2(i-j)+(i-1) \epsilon,
$$

сохранив прежний линейный порядок $s_{1}^{\epsilon}, s_{2}^{\epsilon}, \ldots, s_{n}^{\epsilon}$. Рассмотрим оператор

$$
P_{\lambda}^{(\epsilon)}=\prod_{i<j}^{n} R_{i j}\left(s_{i}^{\epsilon}-s_{j}^{\epsilon}\right),
$$




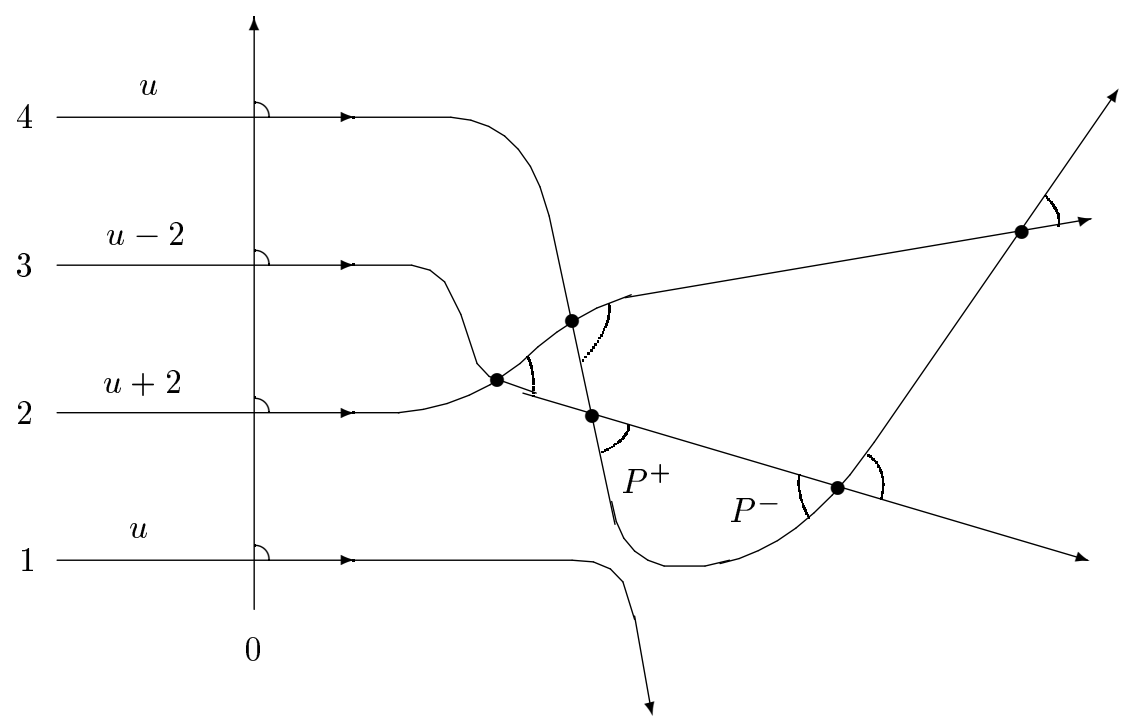

Рис. 7.

где сомножители расставлены в том же порядке, что и в (2.8).

ЛЕмма $2.1[31] . \Pi p u \epsilon \rightarrow 0$

$$
P_{\lambda}^{(\epsilon)}=c \epsilon^{v} P_{\lambda}+O\left(\epsilon^{v+1}\right)
$$

где $v=\#\left\{\left(i_{1}, j_{1}\right),\left(i_{2}, j_{2}\right) \mid i_{1}<i_{2}, i_{1}-j_{1}=i_{2}-j_{2}\right\}$ есть полное число пар клеток на диагоналях, с - некоторый множитель.

Утверждение леммы позволяет свести доказательство к многократному применению уравнения Янга-Бакстера для $R(u)$.

Процедура слияния в квантовом пространстве проводится аналогично. Проделав ее, можно ввести $R$-матрицу $R^{(\lambda)(\mu)}(u)$, действующую в $V^{(\lambda)} \otimes V^{(\mu)}$, где $\lambda, \mu$ - две произвольные диаграммы Юнга. Уравнение Янга-Бакстера в $\mathcal{V}_{1} \otimes \mathcal{V}_{2} \otimes \mathcal{V}_{3}$ приобретает вид

$$
\begin{aligned}
& R_{12}^{(\lambda)(\mu)}\left(u_{1}-u_{2}\right) R_{13}^{(\lambda)}\left(u_{1}-u_{3}\right) R_{23}^{(\mu)}\left(u_{2}-u_{3}\right)= \\
& =R_{23}^{(\mu)}\left(u_{2}-u_{3}\right) R_{13}^{(\lambda)}\left(u_{1}-u_{3}\right) R_{12}^{(\lambda)(\mu)}\left(u_{1}-u_{2}\right),
\end{aligned}
$$

где $\mathcal{V}_{1}=V^{(\lambda)}, \mathcal{V}_{2}=V^{(\mu)}, \mathcal{V}_{3}=\mathbf{C}^{k}$

“Тривиальные” нули $R^{(\lambda)}(u)$. По построению каждый элемент матрицы $R^{(\lambda)}(u)$ есть некоторьй полином по $u$. Оказывается, что из $R^{(\lambda)}(u)$ можно вынести полиномиальный множитель, общий для всех ее элементов. Его корни здесь и названы "тривиальными" нулями $R$-матрицы. Их можно исключить. Для этого используем те же аргументы, что и в доказательстве теоремы 2.1. Легко видеть, что

$$
R^{(\lambda)}\left(u-s_{i}\right)=0, \quad i=2,3, \ldots, n,
$$


так что после вынесения обшего множителя элементы $R$-матрицы становятся полиномами первого порядка. Если $s_{i}=s_{j}$ для $i \neq j$, надо учитывать кратность нулей. Выделяя общий множитель, переопределим

$$
R^{(\lambda)}(u) \rightarrow R^{(\lambda)}(u)\left(\prod_{i=2}^{n} s_{i}\right)^{-1}
$$

Запишем эту формулу для прямоугольных диаграмм Юнга $\lambda=s^{a}$ с $a$ строками длины $s$ каждая:

$$
R^{\left(s^{a}\right)}(u) \rightarrow R^{\left(s^{a}\right)}(u)\left(\prod_{q=0}^{a-1} \prod_{p=1}^{s-1}(u-2 q+2 p)\right)^{-1}\left(\prod_{q=1}^{a-1}(u-2 q)\right)^{-1}
$$

(при $a=1$ отсутствует однократное произведение, при $s=1-$ двойное).

\section{2. Квантовые матрицы монодромии}

$\mathcal{T}$-матрица. Простейшей квантовой матрицей монодромии ( $\mathcal{T}$-матрицей) является упорядоченное произведение $R$-матриц во вспомогательном пространстве $V_{0}=\mathbf{C}^{k}$ векторного представления группы $G L(k)$ :

$$
\mathcal{T}(u)=R_{0 N}\left(u-y_{N}\right) \ldots R_{02}\left(u-y_{2}\right) R_{01}\left(u-y_{1}\right)
$$

Это $k \times k$-матрища с операторными элементами. Они действуют в квантовом пространстве

$$
\bigotimes_{i=1}^{N} V_{i}, \quad V_{i}=\mathbf{C}^{k}
$$

Параметры $y_{i}$ произвольны, иногда их называют бъстротами или неоднородностями в узлах.

Следуюшее сплетающее соотношение является непосредственным следствием (2.3):

$$
R_{12}(u-v) \mathcal{T}_{13}(u) \mathcal{T}_{23}(v)=\mathcal{T}_{23}(v) \mathcal{T}_{13}(u) R_{12}(u-v)
$$

Квантовое пространство здесь - это тензорное произведение

$$
\mathcal{V}_{3}=\bigotimes_{i=1}^{N} V_{i}
$$

Изобразим $\mathcal{T}$-матрицу графически:

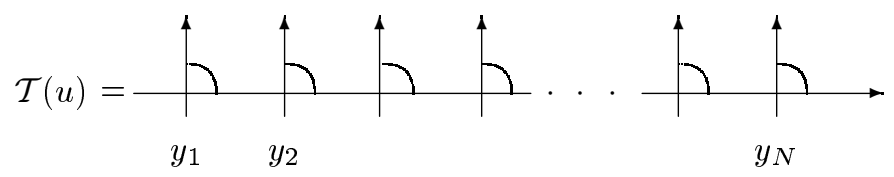


Напомним, что вспомогательное пространство ассоциируется с горизонтальной линией, а квантовое - с вертикальными.

Высшие представления во вспомогательном пространстве. Аналогично (2.16) можно ввести $\mathcal{T}$-матрицу, вспомогательное пространство которой $V_{0}=V^{(\lambda)}$, а квантовое пространство $\bigotimes_{i=1}^{N} V_{i}$ то же, что и раньше:

$$
\mathcal{T}^{(\lambda)}(u)=R_{0 N}^{(\lambda)}\left(u-y_{N}\right) \ldots R_{02}^{(\lambda)}\left(u-y_{2}\right) R_{01}^{(\lambda)}\left(u-y_{1}\right) .
$$

Она удовлетворяет сплетающему соотношению (2.17) с $R$-матрицей, которая получается описанной вьше конструкцией слияния как во вспомогательном, так и в квантовом пространствах.

Каждая $R$-матрица в (2.18) дает вклад в общий полиномиальньй множитель, который можно вынести. Воспользовавшись (2.15), для прямоугольных диаграмм $\lambda=s^{a}$ будем иметь

$$
\mathcal{T}^{\left(s^{a}\right)}(u)=\mathcal{T}_{0}^{\left(s^{a}\right)}(u)\left(\prod_{q=0}^{a-1} \prod_{p=1}^{s-1} \phi(u-2 q+2 p)\right)\left(\prod_{q=1}^{a-1} \phi(u-2 q)\right),
$$

здесь

$$
\phi(u)=\prod_{j=1}^{N}\left(u-y_{j}\right)
$$

$\mathcal{T}_{0}^{\left(s^{a}\right)}(u)$ - ренормированная $\mathcal{T}$-матрица. Если $\phi(u)$ - полином обшего вида, она содержит только нетривиальные “операторные” нули. Если же найдутся такие $i, j$, что $y_{i}-y_{j}=2$, она приобретает дополнительные “тривиальные" нули, которые опять можно исключить. Этот случай реализуется, когда квантовое пространство является тензорным произведеним высших представлений $G L(k)$.

Квантовый детерминант. Максимальная "внешняя степень" фундаментальной $\mathcal{T}$-матрицы (2.16) называется ее квантовым детерминантом. Положим

$$
D^{(k)}(u) \equiv \operatorname{det}_{q} \mathcal{T}(u)=\mathcal{T}^{\left(1^{k}\right)}(u-1+k)
$$

(сдвиг спектрального параметра сделан для удобства в дальнейшем). По определению $D^{(k)}(u)$ - скаляр во вспомогательном пространстве, которое в этом случае одномерно. Следующее утверждение означает, что $D^{(k)}(u)$ является скаляром (т.е. центральным элементом) и в квантовом пространстве.

ТеОрема 2.2. Квантовый детерминант $D^{(k)}(u)$ фундаментальной $\mathcal{T}$-матрицы $\mathcal{T}(u)$ с k-мерным вспомогательным пространством лежит в иентре алгебры, порожденной матричными әлементами $\mathcal{T}$-матрицы:

$$
\left[D^{(k)}(u),(\mathcal{T}(v))_{i j}\right]=0, \quad i, j=1,2, \ldots, k .
$$

Его значение дается явной формулой

$$
D^{(k)}(u)=\phi(u+k+1) \prod_{l=1}^{k-1} \phi(u-k-1+2 l),
$$

где функиия $\phi(u)$ определена в (2.20).

3 Теоретическая и математическая физика, т. 116, № 1, 1998 г. 
ДокАЗАТЕЛЬСтво заключается в использовании уравнения Янга-Бакстера, которое позволяет свести задачу к нахождению квантового детерминанта одной $R$-матрицы, что можно сделать прямым вычислением.

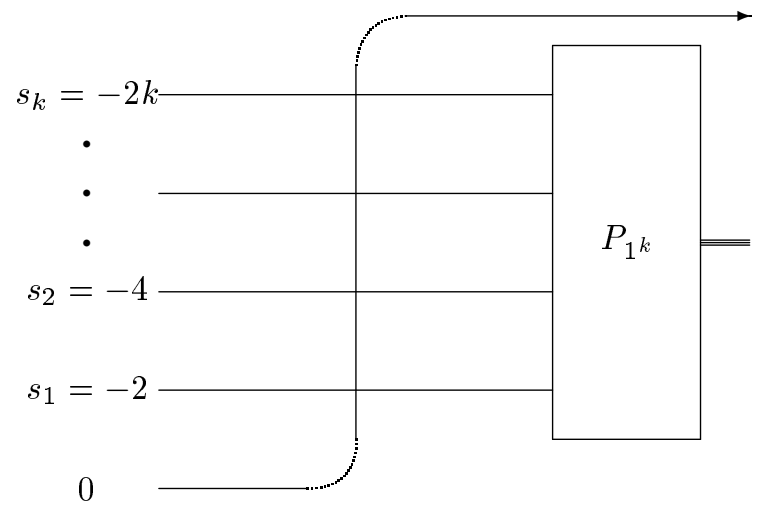

Рис. 8

Покажем, как можно прийти к выражению (2.23) без всяких вычислений. Последние $k-1$ множителей - это в точности нормировка (2.28), так что осталось объяснить лиш первый сомножитель. Заметим, что при $u=2 R$-матрица, участвующая в определении $\mathcal{T}^{\left(1^{k}\right)}(u)$, содержит оператор (изображенный на рис. 8), который тождественно равен нулю, поскольку является проектором на $(k+1)$-ю внешнюю степень пространства $\mathbf{C}^{k}$. Поэтому $R^{\left(1^{k}\right)}(2)=0$. Это и дает первый сомножитель в $(2.23)$.

Понятие квантового детерминанта было введено в работе [32] для квантовых матриц монодромии $2 \times 2$ и обобшено на матрицы $k \times k$ в [23]. Полное доказательство теоремы о явном виде квантового детерминанта, обобщенное на эллиптический случай, можно найти в [33].

\section{3. Квантовые трансфер-матрицы}

Основной объект теории - это квантовая трансфер-матрица ${ }^{2)}$ (T-матрица), которая представляет собой след $\mathcal{T}^{(\lambda)}(u)$ по вспомогательному пространству:

$$
T^{(\lambda)}(u)=\operatorname{tr}_{\text {вспом }} \mathcal{T}^{(\lambda)}\left(u-\lambda_{1}+\lambda_{1}^{\prime}\right),
$$

где $\lambda^{\prime}$ обозначает транспонированную (отраженную относительно главной диагонали) диаграмму, так что $\lambda_{1}^{\prime}$-просто высота первого столбца диаграммы $\lambda$. Сдвиг спектрального параметра сделан для упрошения приведенных дальше формул. В случае прямоугольных диаграмм $s^{a}$ введем специальное обозначение

$$
T_{s}^{a}(u) \equiv T^{\left(s^{a}\right)}(u) .
$$

\footnotetext{
${ }^{2)}$ Название "трансфер-матрица" пришло из статистической механики на решетке. Трансферматрица - это скаляр во вспомогательном пространстве, а слово "матрица" здесь относится к квантовому пространству.
} 
Основное свойство квантовых $T$-матриц - их коммутативность для любых значений непрерывного спектрального параметра $u$ и дискретных параметров $\lambda_{i}$ :

$$
\left[T^{(\lambda)}(u), T^{(\mu)}(v)\right]=0 .
$$

Этот факт следует из уравнения Янга-Бакстера. Таким образом, построено семейство коммутативных операторов в квантовом пространстве. Гамильтониан выбирается так, чтобы он принадлежал этому семейству. Одновременная диагонализация операторов $T^{(\lambda)}(u)$ позволяет полностью описать спектральные свойства квантовой модели.

Заметим, что формулу для квантового детерминанта (2.23) можно переписать следуюшим образом:

$$
T_{1}^{k}(u)=\phi(u+k+1) \prod_{l=1}^{k-1} \phi(u-k-1+2 l) .
$$

Более обшая формула того же типа, которая тоже понадобится в дальнейшем,

$$
T_{s}^{k}(u)=\phi(u+s+k)\left(\prod_{l=0}^{k-1} \prod_{p=1}^{s-1} \phi(u-s-k+2 l+2 p+2)\right) \prod_{l=1}^{k-1} \phi(u-s-k+2 l)
$$

$\left(T_{s}^{0}(u)=1\right)$, выводится с помощью аналогичных рассуждений.

Выделение “тривиальных" нулей (2.19) равносильно изменению нормировки:

$$
T_{s}^{a}(u) \rightarrow T_{s}^{a}(u)\left(\prod_{l=0}^{a-1} \prod_{p=1}^{s-1} \phi(u-s-a+2 l+2 p+2) \prod_{l=1}^{a-1} \phi(u-s-a+2 l)\right)^{-1}
$$

(при $a=1$ произведения по $l$ отсутствуют). Каждое собственное значение $T$-матрицы полином по $u$.

\section{4. Функциональные соотношения и детерминантные формулы}

Между построенными $T$-матрицами имеются многочисленные соотношения, которые в конечном счете следуют из уравнения Янга-Бакстера. Проиллюстрируем механизм их возникновения на самом простом примере. Рассмотрим произведение

$$
T_{1}^{1}(u-2) T_{1}^{1}(u)=\operatorname{Tr}_{V_{1} \otimes V_{2}}\left(\mathcal{T}_{20}(u-2) \otimes \mathcal{T}_{10}(u)\right),
$$

где $V_{1}=V_{2}=\mathbf{C}^{k}$ - вспомогательные пространства, а квантовое пространство $V_{0}$ произвольно. Вставка единичной матрицы $I=P^{+}+P^{-}$, представленной как сумма дополнительных проекторов, и использование циклического свойства следа, свойства проектора $\left(P^{ \pm}\right)^{2}=P^{ \pm}$, а также уравнения Янга-Бакстера приводят к соотношению

$$
T_{1}^{1}(u-2) T_{1}^{1}(u)=T_{1}^{2}(u-1)+T_{2}^{1}(u-1) .
$$

Тем же способом можно вывести более общее соотношение

$$
T_{s}^{1}(u+1) T_{1}^{1}(u-s)=T^{(\lambda=(s, 1))}(u)+T_{s+1}^{1}(u) .
$$

Детальный анализ показывает, что все $T^{(\lambda)}(u)$ могут быть выражены через $T_{s}^{1}(u)$ либо через $T_{1}^{a}(u)$. 
Теорема 2.3 [34]. Справедливы следующие детерминантные формуль:

$$
\begin{aligned}
& T^{(\lambda)}(u)=\operatorname{det}_{1 \leqslant i, j \leqslant \lambda_{1}^{\prime}}\left(T_{\lambda_{i}-i+j}^{1}\left(u-\lambda_{1}^{\prime}+\lambda_{1}-\lambda_{i}+i+j-1\right)\right), \\
& T^{(\lambda)}(u)=\operatorname{det}_{1 \leqslant i, j \leqslant \lambda_{1}}\left(T_{1}^{\lambda_{i}^{\prime}-i+j}\left(u-\lambda_{1}^{\prime}+\lambda_{1}+\lambda_{i}^{\prime}-i-j+1\right)\right),
\end{aligned}
$$

где $T^{(\varnothing)}(u)=1$ (

Здесь подразумевается исходная нормировка без выделения “тривиальных" нулей. Напомним, что $\lambda_{i}^{\prime}$ - длины строк транспонированной диаграммы. Эти формулы были получены Бажановым и Решетихиным [34]. Мы их записали в форме, к которой они были приведены в [35]. Иногда их называют квантовыми тождествами Якоби-Труди. Они представляют собой “янг-бакстеризацию” классических тождеств Якоби-Труди в том смысле, что переходят в последние, если $T$-матрицы не зависят от $u$.

Для прямоугольных диаграмм они упрощаются:

$$
\begin{array}{lll}
T_{s}^{a}(u)=\operatorname{det}\left(T_{s+i-j}^{1}(u-i-j+a+1)\right), & i, j=1, \ldots, a, & T_{s}^{0}(u)=1, \\
T_{s}^{a}(u)=\operatorname{det}\left(T_{1}^{a+i-j}(u-i-j+s+1)\right), & i, j=1, \ldots, s, & T_{0}^{a}(u)=1 .
\end{array}
$$

Во второй формуле подразумевается, что $0<a<k$. Эти красивые детерминантные формулы пока не дают решения основной задачи диагонализации, т.к. лишь сводят ее к определению $T_{s}^{1}(u)$ или $T_{1}^{a}(u)$ (которые входят как “начальные условия").

\section{5. Билинейная форма правил слияния для прямоугольных диаграмм}

Из уравнений (2.31), (2.32) следует, что T-матрицы для прямоугольньи диаграмм Юнга удовлетворяют замкнутой системе функциональных соотношений. Применение тождества Якоби для детерминантов позволяет переписать $(2.31),(2.32)$ в билинейной форме:

$$
T_{s}^{a}(u+1) T_{s}^{a}(u-1)-T_{s+1}^{a}(u) T_{s-1}^{a}(u)=T_{s}^{a+1}(u) T_{s}^{a-1}(u) .
$$

Поскольку все $T_{s}^{a}(u)$ коммутируют, точно такое же соотношение справедливо для каждого из их собственных значений. Поэтому мы можем (и с этого места будем) понимать $T_{s}^{a}(u)$ как численнозначную функцию. В этом соотношении мы узнаем уравнение Хироты (см. формулу (1.3) из [29], в которой надо положить $Z_{1}=-Z_{2}=-Z_{3}=1$ и отождествить $\left.u=x_{1}, s=x_{2}, a=x_{3}, T_{s}^{a}(u)=\tau(u, s, a)\right)$. Отметим, что три переменные $u$, $s, a$, несмотря на их совершенно разное происхождение, вошли в уравнение почти симметричным образом.

Соотношение (2.33) - это вершина абстрактных конструкций КМОЗ в квантовой области. Замечательно, что в этом месте они смыкаются с теорией классических солитонных уравнений.

Итак, мы достигли "вершины” диаграммы (см. рис. 1). Сделаем несколько замечаний. Функциональные соотношения для квантовых $T$-матриц имеют длинную историю. 
Некоторые из них появились в работах $[36,37]$ еше в 1982 г. Общие детерминантные формулы (2.29), (2.30) впервые были указаны в [34]. Билинейная форма была предложена Клюмпером и Пирсом [9] в 1992 г. для моделей $A_{1}$-типа и два года спустя обобшена на модели $A_{k-1}$-типа Кунибой, Наканиши и Сузуки [10]. Они также обобщили ее на модели, ассоциированные с диаграммами Дынкина других серий. В этих работах было продемонстрировано, что функциональные соотношения в билинейной форме, в отличие от детерминантных представлений, способны служить мощным инструментом для практических вычислений в термодинамическом пределе. Кроме того, в работе [10] была сделана попытка их интерпретации с точки зрения теории представлений квантовых аффинных алгебр и янгианов.

Отождествление трансфер-матришы с $\tau$-функцией, билинейных соотношений для нее с уравнением Хироты, а также анализ вытекаюших отсюда следствий (см. ниже) были проведены в наших работах $[17,20]$. Обшие билинейные соотношения для $T$-матрищ, отвечаюших произвольным, а не только прямоугольным диаграммам Юнга, были впервые написаны в [21] (см. также более раннюю работу [18], где приведен один пример таких соотношений); там же они были отождествлены с высшими членами иерархии уравнений Хироты.

\section{6. Об эллиптическом и тригонометрическом случаях}

Все результаты, обзор которых дан в этом разделе, имеют непосредственные аналоги в самой общей ситуации, в которой применим алгебраический аппарат KMO3, - в случае моделей с эллиптической $R$-матрицей. При этом вместо $R$-матришы Янга $(2.2)$ нужно взять эллиптическую $R$-матрицу Белавина [38] с параметрами $\eta$ и $\tau$ :

$$
R(u)=\sum_{a=\left(a_{1}, a_{2}\right) \in \mathbf{Z}_{k}^{2}} \frac{\theta\left[\begin{array}{c}
\frac{a_{1}}{k}+\frac{1}{2} \\
\frac{a_{2}}{k}+\frac{1}{2}
\end{array}\right]\left(\eta\left(u+\frac{2}{k}\right) \mid \tau\right)}{k \theta\left[\begin{array}{c}
\frac{a_{1}}{k}+\frac{1}{2} \\
\frac{a_{2}}{k}+\frac{1}{2}
\end{array}\right]\left(\frac{2 \eta}{k} \mid \tau\right)} \Sigma_{a} \otimes \Sigma_{a}^{-1}
$$

где матрицы

$$
\left(\Sigma_{a}\right)_{i j}=\delta_{i j+a_{1} \bmod k} \exp \left(\frac{2 \pi \sqrt{-1} j a_{2}}{k}\right)
$$

представляют собой аналоги матриц Паули $\sigma_{a}$

$$
\theta\left[\begin{array}{l}
\alpha \\
\beta
\end{array}\right](x \mid \tau)=\sum_{l \in \mathbf{Z}} \exp \left[\pi \sqrt{-1}\left(\tau(l+\alpha)^{2}+2(l+\alpha)(x+\beta)\right)\right]
$$

- $\theta$-функция Якоби с характеристиками. Она обладает свойством $\mathbf{Z}_{k} \times \mathbf{Z}_{k}$-симметрии:

$$
\left(\Sigma_{a} \otimes \Sigma_{a}\right) R(u)\left(\Sigma_{a} \otimes \Sigma_{a}\right)^{-1}=R(u) \quad \forall a \in \mathbf{Z}_{k}^{2},
$$


и следующими свойствами монодромии при сдвиге на периоды:

$$
\begin{aligned}
R\left(u+\frac{1}{\eta}\right)= & -\left(\Sigma_{(0,1)} \otimes I\right)^{-1} R(u)\left(\Sigma_{(0,1)} \otimes I\right) \\
R\left(u+\frac{\tau}{\eta}\right)= & \left(\Sigma_{(1,0)} \otimes I\right)^{-1} R(u)\left(\Sigma_{(1,0)} \otimes I\right) \times \\
& \times \exp \left[-2 \pi \sqrt{-1}\left(u \eta+\frac{2 \eta}{k}+\frac{\tau+1}{2}\right)\right] .
\end{aligned}
$$

Существует также выражение для этой $R$-матрицы через базисные матрицы $E_{i j}$, аналогичное (2.2):

$$
R(u)=\sum R_{i j}^{i^{\prime} j^{\prime}}(u) E_{i i^{\prime}} \otimes E_{j j^{\prime}}
$$

где

$$
R_{i^{\prime} j^{\prime}}^{i j}(u)=\frac{\mathcal{N}(u) \theta\left[\begin{array}{c}
\frac{j-i}{k}+\frac{1}{2} \\
\frac{1}{2}
\end{array}\right](\eta(u+2) \mid k \tau)}{\theta\left[\begin{array}{c}
\frac{i^{\prime}-i}{k}+\frac{1}{2} \\
\frac{1}{2}
\end{array}\right](2 \eta \mid k \tau) \theta\left[\begin{array}{c}
\frac{j-i^{\prime}}{k}+\frac{1}{2} \\
\frac{1}{2}
\end{array}\right](\eta u \mid k \tau)}
$$

при $i+j=i^{\prime}+j^{\prime}(\bmod k)$ и $R_{i^{\prime} j^{\prime}}^{i j}(u)=0$ в противном случае,

$$
\mathcal{N}(u)=\frac{\prod_{l=0}^{k-1} \theta\left[\begin{array}{c}
\frac{l}{k}+\frac{1}{2} \\
\frac{1}{2}
\end{array}\right](\eta u \mid k \tau)}{\prod_{l=1}^{k-1} \theta\left[\begin{array}{c}
\frac{l}{k}+\frac{1}{2} \\
\frac{1}{2}
\end{array}\right](0 \mid k \tau)}
$$

(эта форма $R$-матрицы Белавина взята из работ $[39,40]$ ).

Как и $R$-матрица Янга, $R$-матрица Белавина пропорциональна оператору перестановки при $u=0$ и имеет две точки вырождения $u= \pm 2: R(-2)=U_{-} P^{-}, \quad R(+2)=$ $P^{+} U_{+}$, где $U_{ \pm}-$некоторые обратимые матрицы. Детальный анализ показывает [41], что конструкцию слияния можно провести и в эллиптическом случае. Она опять приводит к детерминантным формулам (2.29), (2.30) и билинейным соотношениям (2.33) того же вида. В формуле (2.27) для квантового детерминанта меняется только вид функции $\phi(u)$ :

$$
\begin{aligned}
& \phi(u)=\prod_{j=1}^{N} \theta_{1}\left(\eta\left(u-y_{j}\right) \mid \tau\right) \\
& \left(\text { здесь } \quad \theta\left[\begin{array}{c}
\frac{1}{2} \\
\frac{1}{2}
\end{array}\right](u \mid \tau) \equiv \theta_{1}(u \mid \tau)\right) \text {. }
\end{aligned}
$$

Мы будем назьвать функции этого вида эллиптическими полиномами от $u$, а число $N$ - степенью эллиптического полинома. Эллиптические полиномы степени $N$ характеризуются свойствами монодромии

$$
\begin{aligned}
\phi\left(u+\frac{1}{\eta}\right) & =(-1)^{N} \phi(u) \\
\phi\left(u+\frac{\tau}{\eta}\right) & =\exp \left(-N \pi i \tau-2 \pi i \eta\left(N u-\sum_{j=1}^{N} y_{i}\right)\right) \phi(u)
\end{aligned}
$$


при сдвиге на периоды. Их часто называют также $\theta$-функциями порядка $N$. Заметим, что в силу свойств $(2.36),(2.37)$ матричные элементы трансфер-матриц являются эллиптическими полиномами от $u$, взятие следа от произведения $R$-матрищ по первому пространству дает закон преобразования при сдвиге на периоды, идентичный (2.41).

В предельных случаях $\tau=\infty$ или $\tau=0 R$-матрица Белавина вырождается в тригонометрическую:

$$
\begin{aligned}
& R_{i^{\prime} j^{\prime}}^{i j}(u)=\delta_{i j} \delta_{i i^{\prime}} \delta_{i^{\prime} j^{\prime}} \sin \pi \eta(u+2)+ \\
& \quad+\delta_{i i^{\prime}} \delta_{j j^{\prime}}\left(1-\delta_{i^{\prime} j^{\prime}}\right) \sin \pi \eta u \exp \left[\frac{2 \pi \sqrt{-1} \eta}{k}(2(j-i)-k \operatorname{sign}(j-i))\right]+ \\
& \quad+\delta_{i j^{\prime}} \delta_{i^{\prime} j}\left(1-\delta_{i^{\prime} j^{\prime}}\right) \sin 2 \pi \eta \exp \left[-\frac{\pi \sqrt{-1} u}{k}(2(j-i)-k \operatorname{sign}(j-i))\right]
\end{aligned}
$$

Все функциональные соотношения остаются в силе, а функция $\phi(u)$ становится “тригонометрическим полиномом" степени $N$ :

$$
\phi(u)=\prod_{j=1}^{N} \sin \pi \eta\left(u-y_{j}\right)
$$

(т.е. полиномом Лорана от $e^{i \pi \eta u}$ ).

Эллиптическое (2.38) и тригонометрическое (2.42) решения уравнения Янга-Бакстера уже не обладают свойством $G L(k)$-инвариантности, но по-прежнему в очевидном смысле ассоциированы с системой корней $A_{k-1}$. Квантовые матрицы монодромии, построенные из таких $R$-матриц, образуют представления обобшенных алгебр Склянина $[42,43]$ или квантовых алгебр $U_{q}\left(s l_{k}\right)$. Для краткости мы будем назьвать интегрируемые модели с $R$-матрицей $\left(k^{2} \times k^{2}\right)$ Белавина $(2.38)$ или ее вырождениями - моделями $A_{k-1}$-типа или просто $A_{k-1}$-моделями .

\section{3. СВОЙСТВА РЕШЕНИЙ УРАВНЕНИЯ ХИРОТЫ, ОТВЕЧАЮШИХ КВАНТОВОЙ ЗАДАЧЕ}

Начиная с этого раздела мы приступаем к систематическому изучению билинейных функциональных соотношений для трансфер-матриц, которые для краткости иногда будем называть правилами слияния. Билинейная форма, которую для удобства ссылок приведем здесь еше раз,

$$
T_{s}^{a}(u+1) T_{s}^{a}(u-1)-T_{s+1}^{a}(u) T_{s-1}^{a}(u)=T_{s}^{a+1}(u) T_{s}^{a-1}(u)
$$

оказывается гораздо более полезной, чем детерминантные формулы $(2.29),(2.30)$, поскольку позволяет получить полное решение задачи, а не только свести ее к нахождению $T_{s}^{1}(u)$ или $T_{1}^{a}(u)$. В то же время эта форма указывает на глубокую связь с теорией классических солитонных уравнений. Поэтому мы изменим точку зрения и на время "забудем" очерченный выше вывод уравнения (3.1) из первых принципов квантовой теории, 
посмотрев на (3.1) как на основное уравнение, управляющее спектральными свойствами системы, и попытавшись извлечь из него следствия. Тем самым будет осуществлен поэтапный “спуск” с вершины. Иными словами, мы собираемся воспроизвести основные результаты анзаца Бете путем решения уравнения (3.1) методами, естественными для теории классических интегрируемых уравнений.

Уравнение Хироты само по себе имеет огромное количество решений самой разной природы. Наша ближайшая цель - охарактеризовать тот класс решений, в который попадают собственные значения трансфер-матриц. Для этого надо фиксировать граничные условия и аналитические свойства решений.

\section{1. Граничные условия по $a$ и $s$}

Функции $T_{s}^{0}(u)$ и $T_{s}^{k}(u)$ для $A_{k-1}$-моделей нужно рассматривать как граничные условия. Напомним, что в исходной нормировке $T_{s}^{0}(u)=1$, а $T_{s}^{k}(u)$ равна квантовому детерминанту (2.27):

$$
T_{s}^{k}(u)=\prod_{p=0}^{s-1}\left(\phi(u-s+k+2+2 p) \prod_{l=1}^{k-1} \phi(u-s-k+2 p+2 l)\right)
$$

Заметим, что $T_{s}^{k}(u)$ удовлетворяет дискретному уравнению Д'Аламбера

$$
T_{s}^{k}(u+1) T_{s}^{k}(u-1)=T_{s+1}^{k}(u) T_{s-1}^{k}(u) .
$$

Обшее решение дискретного уравнения Д'Аламбера можно представить в виде $\chi_{+}(u+s) \chi_{-}(u-s)$, т.е. оно факторизуется в произведение "голоморфной” $\chi_{+}$и “антиголоморфной" функции $\chi_{-}$. Покажем, как представить $T_{s}^{k}(u)$ в этой форме. Например, в рациональном случае имеем

$$
\begin{gathered}
T_{s}^{k}(u)=\frac{\chi_{k}(u+s)}{\chi_{k}(u-s)} \\
\chi_{k}(u)=2^{N k u / 2} \prod_{i=1}^{N}\left(\Gamma\left(\frac{u+k-y_{i}}{2}+1\right) \prod_{l=1}^{k-1} \Gamma\left(\frac{u-k-y_{i}}{2}+l\right)\right),
\end{gathered}
$$

где $\Gamma(u)$ - гамма-функция. Граничное значение на левом конце $T_{s}^{0}(u)=1$, очевидно, тоже удовлетворяет дискретному уравнению Д'Аламбера. Эти свойства не противоречат уравнению Хироты только при $T_{s}^{-1}(u)=T_{s}^{-2}(u)=0$ и $T_{s}^{k+1}(u)=T_{s}^{k+2}(u)=0$, а на $T_{s}^{a}(u)$ при $a \leqslant-3$ и $a \geqslant k+3$ никаких ограничений не возникает. Доопределим граничные условия следуюшим образом:

$$
T_{s}^{a}(u)=0 \quad \text { при } \quad a<0 \quad \text { или } \quad a>k .
$$

С точки зрения теоретико-группового подхода индекс $a$ пробегает вершины граф̆а Дынкина. 
Перейдем к граничным условиям по $s$. Из $T_{0}^{a}(u)=1$ при $0<a<k$ сразу следует, что $T_{-1}^{a}(u)=0$ при $0<a<k$. Выписав цепочку уравнений Хироты для $-k \leqslant s \leqslant 0$, можно показать, что полное граничное условие по $s$ имеет вид

$$
T_{s}^{a}(u)=0 \quad \text { при } \quad-k<s<0 \quad \text { и } \quad 0<a<k,
$$

причем остальные функции $T_{s}^{a}(u)$ при $0<a<k$, вообще говоря, не равны тождественно нулю. Его смысл будет обсуждаться ниже.

\section{2. Аналитические условия по $u$}

Для моделей на конечных решетках с эллиптической $R$-матрицей функция $T_{s}^{a}(u)$ при любых фиксированных $a, s$ должна быть эллиптическим полиномом по спектральному параметру $u$. Как уже отмечалось, из закона преобразования $(2.36),(2.37) R$-матришы Белавина при сдвигах на периоды и конструкции квантовой матришы монодромии следует, что все матричные элементы этих операторов - следов $\mathcal{T}$-матриц по первому пространству - суть эллиптические полиномы по $u$. Вместе с тем они коммутируют при всех $и$ и поэтому могут быть одновременно приведены к диагональному виду преобразованием сопряжения, не зависяшим от $u$. Отсюда следует, что их собственные значения тоже эллиптические полиномы от $u$ :

$$
T_{s}^{a}(u)=A_{s}^{a} \prod_{j=1}^{N(a, s)} \theta_{1}\left(\eta\left(u-w_{j}^{(a, s)}\right)\right),
$$

где $w_{j}^{(a, s)}, A_{s}^{a}$ не зависят от $u$.

При таком виде $T_{s}^{a}(u)$ равенство в (3.1) может иметь место, только если все три слагаемых одинаково преобразуются при сдвиге на периоды. Отсюда на сумму корней

$$
W^{(a, s)}=\sum_{j=1}^{N(a, s)} w^{(a, s)}
$$

накладываются условия связи

$$
W^{(a, s+1)}+W^{(a, s-1)}=W^{(a+1, s)}+W^{(a-1, s)}=2 W^{(a, s)}
$$

по модулю сдвига по решетке периодов и на их число

$$
N(a, s+1)+N(a, s-1)=N(a+1, s)+N(a-1, s)=2 N(a, s) .
$$

Общее решение этих уравнений - функция от $a, s$ вида $C_{1} a s+C_{2} a+C_{3} s+C_{4}$ с произвольными константами $C_{i}$. Эти условия можно сформулировать иначе, сказав, что "калибровочно-инвариантные" величины

$$
\frac{T_{s+1}^{a}(u) T_{s-1}^{a}(u)}{T_{s}^{a}(u+1) T_{s}^{a}(u-1)}, \quad \frac{T_{s}^{a+1}(u) T_{s}^{a-1}(u)}{T_{s}^{a}(u+1) T_{s}^{a}(u-1)}
$$

являются эллиптическими функциями от $u$.

Для моделей с рациональной (тригонометрической) $R$-матрицей $T_{s}^{a}(u)$ вырождается в обычный (соответственно тригонометрический) полином. 


\section{3. Нормировка}

Из (3.1) непосредственно видно, что $T_{s}^{a}(u)$ можно умножать на "калибровочные" множители

$$
\chi_{0}(u+s+a) \chi_{1}(u+s-a) \chi_{2}(u-s+a) \chi_{3}(u-s-a)
$$

с произвольными функциями $\chi_{i}$, оставляя вид уравнения неизменным. Граничные и аналитические условия могут поэтому зависеть от выбора “калибровки" (нормировки). Наиболее употребительными являются следующие три специальных способа нормировки.

Детерминантная нормировка. Она уже использовалась в предыдушем разделе. В этой нормировке $T_{s}^{0}(u)=1$, а $T_{s}^{k}(u)$ равняется квантовому детерминанту (3.2). Степень полиномов $T_{s}^{a}(u)$ растет с увеличением $a$ и $s$.

Каноническая нормировка. Поскольку функции $T_{s}^{0}(u)$ и $T_{s}^{k}(u)$ на границах удовлетворяют дискретному уравнению Д'Аламбера, умножением на “калибровочные" множители их можно сделать равными единице: $T_{s}^{0}(u)=T_{s}^{k}(u)=1$. Это позволяет упростить уравнения ценой усложнения аналитических свойств их решений.

Минимальная нормировка. Указанное выше "калибровочное" преобразование позволяет сократить все нули на характеристиках $a \pm s \pm u=$ const. Заметим, что это как раз “тривиальные” нули $T$-матрицы, своим происхождением обязанные процедуре “слияния". Сократив их, можно уменьшить степень полиномов $T_{s}^{a}(u)$. Полиномы минимально возможной степени возникают в нормировке (2.28). На границах (т.е. при $a=0, k)$ они принимают вид

$$
T_{s}^{0}(u)=\phi(u-s), \quad T_{s}^{k}(u)=\phi(u+s+k)
$$

(напомним, что $\phi(u)$ - эллиптический полином степени $N(2.40)$ ). Отметим, что в этой нормировке $T^{0}{ }_{-}$“антиголоморфная” функция (т.е. она зависит только от $\left.u-s\right)$, а $T^{k}$ “голоморфная" (зависит только от $u+s$ ). Другое удобное свойство минимальной нормировки состоит в том, что эллиптические полиномы $T_{s}^{a}(u)$ имеют одну и ту же степень $N(a, s)=N$ при всех значениях $a, s$ (если только $T_{s}^{a}(u)$ не равно тождественно нулю). Отметим, что в минимальной нормировке из (3.8) следует

$$
T_{0}^{a}(u)=\phi(u+a) \quad \text { при } \quad 0 \leqslant a \leqslant k .
$$

В дальнейшем мы используем минимальную нормировку.

Теперь можно уточнить постановку задачи. Функция $\phi(u)$ считается заданной в виде эллиптического, тригонометрического или обычного полинома ${ }^{3)}$, и требуется найти все решения $T_{s}^{a}(u)$ уравнения Хироты с указанными выше граничными условиями в виде полинома той же степени.

\footnotetext{
3) Если нет специальных уточнений, ниже под словом полином понимается функция любого из этих трех видов.
} 


\section{4. Обсуждение}

Обсудим наложенные условия и подведем некоторые итоги.

Уравнение (3.1) с граничными условиями (3.5) известно как дискретный аналог так называемой двумеризованной молекулы Тоды в билинейной форме [44] - двумеризованной цепочки Тоды с открытыми концами. Отметим, что ее можно рассматривать как интегрируемую дискретизацию конформной полевой теории Тоды [45]. В частности, при $k=2$ получаем дискретный аналог уравнения Лиувилля.

Граничное условие по $s$ вида (3.6) тоже не раз возникало в классической теории. Условия этого типа (тождественное обрашение $\tau$-функции в нуль при одном из значений дискретной переменной) - характерный признак "полубесконечныц" ( "forced") uерар$x и и ̆$ нелинейных интегрируемых уравнений, естественно возникаюших в матричных моделях двумерной гравитации (см., например, [46]).

Наконец, наложение аналитических условий типа (3.7) на $\tau$-функцию - тоже знакомый сюжет в теории солитонных уравнений. Исследование их әллиптических рещений, а именно в этот класс и попадают интересуюшие нас решения вида (3.7), берет свое начало с работы [47], в которой были исследованы эллиптические решения уравнения Кортевега-де Фриза. Систематический подход к изучению эллиптических решений более обших уравнений, основанный на технике конечнозонного интегрирования, был развит Кричевером [48]. Об эллиптических решениях разностных солитонных уравнений см. [49].

Таким образом, мы видим, что каждое из наложенных выше условий хорошо известно в классической теории. Однако до сих пор они никогда не встречались вместе в одной и той же задаче. По-видимому, вся специфика решений, описываюших квантовые системы, состоит как раз в комбинации этих трех условий, которая выглядит достаточно необычно с точки зрения теории солитонных уравнений.

\section{4. ИЕРАРХИЧЕСКИЙ АНЗАЦ БЕТЕ КАК ЦЕПОЧКА ПРЕОБРАЗОВАНИЙ БЕКЛУНДА}

\section{1. Представление нулевой кривизны и вспомогательные линейные задачи}

Теперь можно применить к уравнению (3.1) методы классической теории. Первое, что можно сделать, - это представить его как условие нулевой кривизны или, эквивалентно, как условие коммутации некоторых разностных операторов. Сделаем необходимые для этого приготовления.

Пользуясь терминологией работы [29], выберем $a$ в качестве базисной переменной. Это означает, что разностные операторы, участвуюшие в представлении нулевой кривизны, будут действовать на функции от $a$. Далее, от “лабораторных" переменных $u, s$ перейдем к "переменным светового конуса" $l, \bar{l}$, положив

$$
l=\frac{1}{2}(u-s-a), \quad \bar{l}=\frac{1}{2}(-u-s+a),
$$


т.е.

$$
s=-l-\bar{l}, \quad u=l-\bar{l}+a .
$$

Введем также функцию

$$
T^{a}(l, \bar{l}) \equiv T_{s}^{a}(u),
$$

где переменные связаны вышеприведенными формулами. Уравнение Хироты примет вид

$$
T^{a}(l+1, \bar{l}) T^{a}(l, \bar{l}+1)-T^{a}(l, \bar{l}) T^{a}(l+1, \bar{l}+1)=T^{a-1}(l+1, \bar{l}) T^{a+1}(l, \bar{l}+1)
$$

с условием

$$
T^{a}(l, \bar{l})=0 \text { при всех } a \text {, кроме } 0 \leqslant a \leqslant k .
$$

Рассмотрим разностные операторы

$$
\begin{aligned}
& \mathcal{M}=e^{\partial_{a}}+\frac{T^{a}(l, \bar{l}) T^{a+1}(l+1, \bar{l})}{T^{a}(l+1, \bar{l}) T^{a+1}(l, \bar{l})}, \\
& \overline{\mathcal{M}}=1+\frac{T^{a+1}(l, \bar{l}+1) T^{a-1}(l, \bar{l})}{T^{a}(l, \bar{l}+1) T^{a}(l, \bar{l})} e^{-\partial_{a},}
\end{aligned}
$$

вид которых прямо следует из формул работы [29]. Уравнение (4.1) эквивалентно разностному аналогу условия нулевой кривизны

$$
\overline{\mathcal{M}}(l+1, \bar{l}) \mathcal{M}(l, \bar{l})=\mathcal{M}(l, \bar{l}+1) \overline{\mathcal{M}}(l, \bar{l}) .
$$

Вспомогательные линейные задачи (ВЛЗ) имеют вид

$$
\begin{aligned}
\left(e^{\partial_{a}}+\frac{T^{a}(l, \bar{l}) T^{a+1}(l+1, \bar{l})}{T^{a}(l+1, \bar{l}) T^{a+1}(l, \bar{l})}\right) \Psi & =e^{\partial_{l}} \Psi, \\
\left(1+\frac{T^{a+1}(l, \bar{l}+1) T^{a-1}(l, \bar{l})}{T^{a}(l, \bar{l}+1) T^{a}(l, \bar{l})} e^{-\partial_{a}}\right) \Psi & =e^{\partial_{\bar{l}}} \Psi .
\end{aligned}
$$

Поскольку $T^{a}(l, \bar{l})$ может обрашаться в нуль, имеет смысл освободиться от знаменателей, введя функцию

$$
F^{a}(l, \bar{l})=\Psi^{a}(l, \bar{l}) T^{a}(l, \bar{l}),
$$

в терминах которой будем иметь

$$
\begin{aligned}
& T^{a}(l+1, \bar{l}) F^{a+1}(l, \bar{l})+T^{a+1}(l+1, \bar{l}) F^{a}(l, \bar{l})=T^{a+1}(l, \bar{l}) F^{a}(l+1, \bar{l}), \\
& T^{a}(l, \bar{l}+1) F^{a}(l, \bar{l})+T^{a+1}(l, \bar{l}+1) F^{a-1}(l, \bar{l})=T^{a}(l, \bar{l}) F^{a}(l, \bar{l}+1) .
\end{aligned}
$$

Преимушество "переменных светового конуса" в том, что они разделяются в ВЛЗ. Однако, в отличие от $a, s, u$, они не имеют непосредственного физического смысла. После того как представление нулевой кривизны написано, не составляет труда переписать 
его в “лабораторных" переменных. Из (4.3) сразу получаем коммутационное представление $[\mathfrak{M}, \overline{\mathfrak{M}}]=0$, где

$$
\begin{aligned}
& \mathfrak{M}=e^{\partial_{s}-\partial_{u}}\left(e^{\partial_{a}+\partial_{u}}+\frac{T_{s}^{a}(u) T_{s-1}^{a+1}(u+2)}{T_{s-1}^{a}(u+1) T_{s}^{a+1}(u+1)}\right), \\
& \overline{\mathfrak{M}}=e^{\partial_{s}+\partial_{u}}\left(1+\frac{T_{s-1}^{a+1}(u) T_{s}^{a-1}(u-1)}{T_{s-1}^{a}(u-1) T_{s}^{a}(u)} e^{-\partial_{a}-\partial_{u}}\right) .
\end{aligned}
$$

Формулы (4.6) примут вид

$$
\begin{aligned}
T_{s+1}^{a+1}(u) F_{s}^{a}(u)-T_{s}^{a+1}(u+1) F_{s+1}^{a}(u-1) & =T_{s}^{a}(u) F_{s+1}^{a+1}(u), \\
T_{s+1}^{a}(u+1) F_{s}^{a}(u)-T_{s}^{a}(u) F_{s+1}^{a}(u+1) & =T_{s}^{a+1}(u+1) F_{s+1}^{a-1}(u),
\end{aligned}
$$

где $F_{s}^{a}(u) \equiv F^{a}(l, \bar{l})$. С учетом того, что $T$-функции на гранишах удовлетворяют дискретному уравнению Д'Аламбера (3.3), совместность этих ВЛЗ эквивалентна уравнению Хироты (2.33).

Из (4.9) следует, что функция $F_{s}^{a}(u)$ удовлетворяет уравнению Хироты точно такого же вида ${ }^{4)}$

$$
F_{s}^{a}(u+1) F_{s}^{a}(u-1)-F_{s+1}^{a}(u) F_{s-1}^{a}(u)=F_{s}^{a+1}(u) F_{s}^{a-1}(u) .
$$

Естественно потребовать, чтобы $F_{s}^{a}(u)$ имела те же аналитические свойства по $u$, что и $T_{s}^{a}(u)$ (хотя степень эллиптического полинома может быть другой).

Решение $F$ допускает несколько разных граничных условий. Среди них имеется одно, аналогичное (3.5), но с на единицу меньшим числом ненулевых функций $F$ :

$$
F_{s}^{a}(u)=0 \quad \text { при } \quad a<0 \quad \text { или } \quad a>k-1 .
$$

Функции $F^{a}$ на краях диаграммы Дынкина $(a=0, k-1)$ имеют очень специальный вид. Из второго уравнения (4.9) при $a=0$ и из первого при $a=k-1$ следует, что $F_{s}^{0}(u)$ (соответственно $F_{s}^{k-1}(u)$ ) зависит только от $u-s$ (соответственно от $u+s$ ). Введем для них специальные обозначения:

$$
F_{s}^{0}(u)=Q_{k-1}(u-s), \quad F_{s}^{k-1}(u)=\bar{Q}_{k-1}(u+s) .
$$

Далее, можно показать, что требование (3.8), специфическое для решений, описывающих квантовые системы, позволяет наложить аналогичное условие, которое связывает функции $Q_{k-1}$ и $\bar{Q}_{k-1}$ :

$$
\bar{Q}_{k-1}(u)=Q_{k-1}(u+k-1) .
$$

Переход от $T$ к $F$ - это преобразование типа Беклунда. Аналитические свойства и граничные условия для $F_{s}^{a}(u)$ такие же, как для $T_{s}^{a}(u)$, если вместо $\phi(u)$ подставить $Q_{k-1}(u)$ и учесть сокрашение длины диаграммы Дьнкина: $k \rightarrow k-1$. Повторяя эту процедуру, можно последовательно свести $A_{k-1}$-модель к $A_{1}$-модели. Ниже это замечание будет использовано для вьвода уравнений иерархического $A_{k-1}$-анзаца Бете.

\footnotetext{
4) В работе [50] этот факт был назван свойством дуальности между "потенциалом" и "волновой функцией”, см. также [29].
} 


\section{2. Функции $Q_{t}(u)$}

Для анализа цепочки преобразований Беклунда можно ввести новую дискретную переменную $t$, нумерующую последовательные шаги “потока" $A_{k-1} \rightarrow A_{k-2} \rightarrow \cdots \rightarrow A_{1}$ в обратном направлении. Именно, пусть $T_{s, k-t}^{a}(u)$ - решение линейных задач на $t$-м шаге. В этих обозначениях $T_{s, k}^{a}(u)=T_{s}^{a}(u)$ и $T_{s, k-1}^{a}(u)=F_{s}^{a}(u)$. Далее, на любом "уровне" $t$ функция $T_{s, t}^{a}(u)$ удовлетворяет уравнению Хироты (4.10) с граничными условиями

$$
T_{s, t}^{a}(u)=0 \quad \text { при } \quad a<0 \quad \text { или } \quad a>t .
$$

Первый $(a=0)$ и последний $(a=t)$ члены этой последовательности удовлетворяют дискретному уравнению Д'Аламбера и являются соответственно “антиголоморфной” и “голоморфной” функциями. Введем для них специальные обозначения:

$$
T_{s, t}^{0}(u) \equiv Q_{t}(u-s), \quad T_{s, t}^{t}(u) \equiv \bar{Q}_{t}(u+s)
$$

обобщающие (4.12). Их можно распространить и на $t=k$, положив

$$
Q_{k}(u)=\phi(u), \quad \bar{Q}_{k}(u)=\phi(u+k) .
$$

То, что $\bar{Q}_{k}$ - зависимая от $Q_{k}$ функция и отличается от нее только сдвигом аргумента, является специфическим требованием, вытекающим из постановки квантовой задачи на конечной решетке. Эту связь можно непротиворечиво продолжить на всю последовательность функций $Q_{t}$ для $1 \leqslant t \leqslant k$ :

$$
\bar{Q}_{t}(u)=Q_{t}(u+t)
$$

Линейные задачи (4.9) на уровне $t$ :

$$
\begin{aligned}
& T_{s+1, t+1}^{a+1}(u) T_{s, t}^{a}(u)-T_{s, t+1}^{a+1}(u+1) T_{s+1, t}^{a}(u-1)=T_{s, t+1}^{a}(u) T_{s+1, t}^{a+1}(u), \\
& T_{s+1, t+1}^{a}(u+1) T_{s, t}^{a}(u)-T_{s, t+1}^{a}(u) T_{s+1, t}^{a}(u+1)=T_{s, t+1}^{a+1}(u+1) T_{s+1, t}^{a-1}(u),
\end{aligned}
$$

выглядят теперь как билинейные уравнения на функцию от четырех переменных. Однако уравнение (4.17) (соответственно (4.18)) можно ограничить на гиперплоскость $u+s-a=$ const (соответственно $u-s-a=$ const), при этом четвертая переменная войдет как параметр.

Ограничив (4.17) на гиперплоскость $u+s-a=0$, т.е. положив

$$
f_{u}(t, a) \equiv T_{a-u, k-t}^{a}(u),
$$

мы сведем это уравнение к уравнению Хироты для $f_{u}(t, a)$

$$
f_{u}(t+1, a) f_{u}(t, a+1)-f_{u}(t, a) f_{u}(t+1, a+1)=f_{u-1}(t+1, a) f_{u+1}(t, a+1),
$$


в котором в отличие от (3.1) $t$ и $а$ входят как переменные светового конуса. Граничные условия следующие:

$$
f_{u}(t, 0)=Q_{k-t}(2 u), \quad f_{u}(t, k-t)=\bar{Q}_{k-t}(t-k)=\text { const } .
$$

Аналогичное уравнение следует и из второй линейной задачи (4.18).

Всю эту совокупность функций удобно изобразить на диаграмме; приведем пример для $A_{3}$-случая $(k=4)$ :

$$
\begin{aligned}
& 0 \quad 1 \quad 0 \\
& 0 \quad Q_{1}(u-s) \quad \bar{Q}_{1}(u+s) \quad 0 \\
& \begin{array}{lllll}
0 & Q_{2}(u-s) & T_{s, 2}^{1}(u) & \bar{Q}_{2}(u+s) & 0
\end{array} \\
& \begin{array}{llllll}
0 & Q_{3}(u-s) & T_{s, 3}^{1}(u) & T_{s, 3}^{2}(u) & \bar{Q}_{3}(u+s) & 0
\end{array} \\
& \begin{array}{lllllll}
0 & Q_{4}(u-s) & T_{s, 4}^{1}(u) & T_{s, 4}^{2}(u) & T_{s, 4}^{3}(u) & \bar{Q}_{4}(u+s) & 0 .
\end{array}
\end{aligned}
$$

Функции в каждом горизонтальном сечении с постоянным $t$ удовлетворяют уравнению Хироты (4.10). Уравнение Хироты для беклундовского потока в переменных светового конуса (4.20) действует, условно говоря, “по вертикали”.

В иерархическом анзаце Бете функции $T_{s, t}^{a}(u)$ при $t<k$ являются вспомогательными объектами; по смыслу они совпадают с собственными значениями $T$-матриц для “промежуточных" моделей, возникаюших на $(k-t)$-м шаге (уровне) иерархического анзаца Бете ${ }^{5)}$. Функции $Q_{t}(u)$, хотя тоже не имеют непосредственного физического смысла, играют очень важную роль. Их можно отождествить с собственными значениями обобщенных $Q$-операторов Бакстера (см. ниже). В общем решении уравнения Хироты они выступают как произвольные функциональные параметры. Требование эллиптичности определяет их через систему уравнений Бете на их нули. В следующем разделе будет введен другой набор функциональных параметров, которые остаются свободными даже после наложения условия эллиптичности. В конце этого раздела мы покажем, что динамика нулей функций $Q_{t}$ описывается уравнениями иерархического анзаца Бете.

\section{3. Уравнения Бете как динамическая система в дискретном времени}

Напомним, что функция $f_{u}(t, a)(4.19)$ удовлетворяет уравнению Хироты в форме (4.20). Заметим, что если отвлечься от граничного условия, оно буквально совпадает с (4.1) после замены $a$ на $u$ и отождествления $f_{u}(t, a)=T^{u}(t, a)$. Поставим задачу о нахождении эллиптических решений уравнения (4.20), т.е. таких, в которых $f_{u}(t, a)$ является эллиптическим полиномом по $u$ при всех значениях $t, a$. В частности, при $a=0$ имеем $f_{u}(t, 0)=Q_{k-t}(2 u)$, т.е., решив эту задачу, мы определим, как нули функции $Q_{t}$ зависят от $t$. Какое отношение эта задача имеет к исходной - нахождению эллиптических решений уравнения (3.1) - и как позволяет ее решить, будет обсуждаться в следующем разделе.

\footnotetext{
5) В отличие от (3.1) уравнениям (4.17), (4.18) нельзя придать операторный смысл, поскольку $T_{s, t}^{a}(u)$ и $T_{s, t+1}^{a}(u)$ - собственные значения операторов, действующих в разных квантовых пространствах.
} 
Пользуясь понятиями из [29], поясним, в чем заключается принципиальная разница в постановке задачи об эллиптических по $u$ решениях уравнений (3.1) и (4.20). В первом случае поток $u$ составной, и мы интересуемся динамикой нулей выражения (3.7) по другому составному потоку. Во втором случае поток $u$ элементарный, и мы будем изучать динамику нулей функции $Q_{k-t}(2 u)$ в (4.21) по другому элементарному потоку, к нему примыкаюшему. Метод нахождения эллиптических решений, развитый Кричевером [48] первоначально для уравнения Кадомцева-Петвиашвили, заключается в обращении к ВЛЗ с базисной переменной $u$. В каждой такой ВЛЗ, в отличие от нелинейного уравнения, переменные разделены с самого начала, что позволяет найти динамику нулей $\tau$-функции по каждому потоку независимо. Отсюда ясно, что вторая задача проше, чем первая. В самом деле, в первом случае базисный поток составной, и, следовательно, простейшие возможные ВЛЗ, в которых переменные разделяются, имеют матричную форму. Во второй же задаче можно обойтись трехчленными уравнениями со скалярными коэффициентами.

Проблема нахождения эллиптических решений (т.е. описание динамики нулей $\tau$-функции или полюсов отношений $\tau$-функций) в такой постановке стала стандартной после работ $[48,51,49]$. Ее решение обнаруживает замечательные связи с системой частиц Калоджеро-Мозера или Рейсенарса ${ }^{6)}$ и, как мы вскоре убедимся, с уравнениями иерархического анзаца Бете.

Итак, приступим к выводу уравнений, описьвающих динамику нулей функции

$$
f_{u}(t, 0)=Q_{k-t}(2 u)
$$

по $t$. Предположим, что $Q_{t}(u)$ имеет вид

$$
Q_{t}(u)=\prod_{j=1}^{M_{t}} \theta_{1}\left(\eta\left(u-u_{j}^{t}\right)\right) .
$$

Заметим, что число корней $M_{t}$ может зависеть от $t$. Сами уравнения движения (без представления Лакса) выводятся элементарно [49]. Рассмотрим ВЛЗ для уравнения (4.20) вида (4.6). Так как мы интересуемся динамикой корней по $t$ при фиксированном $a=0$, достаточно рассмотреть только первое линейное уравнение, которое при $a=0$ примет вид

$$
Q_{k-t-1}(2 u) g^{t}(u+1)+Q_{k-t-1}(2 u+2) g^{t}(u)=Q_{k-t}(2 u+2) g^{t+1}(u),
$$

где мы положили $g^{t}(u) \equiv F^{u}(t, 0)$. Положим $2 u$ по очереди равным $u_{j}^{k-t-1}, u_{j}^{k-t-1}-2$ и $u_{j}^{k-t}-2$, чтобы в этом уравнении оставалось только два члена:

$$
\begin{aligned}
Q_{k-t-1}\left(u_{j}^{k-t-1}+2\right) g^{t}\left(u_{j}^{k-t-1}\right) & =Q_{k-t}\left(u_{j}^{k-t-1}+2\right) g^{t+1}\left(u_{j}^{k-t-1}\right), \\
Q_{k-t-1}\left(u_{j}^{k-t-1}-2\right) g^{t}\left(u_{j}^{k-t-1}\right) & =Q_{k-t}\left(u_{j}^{k-t-1}\right) g^{t+1}\left(u_{j}^{k-t-1}-2\right), \\
Q_{k-t-1}\left(u_{j}^{k-t}-2\right) g^{t}\left(u_{j}^{k-t}\right) & =-Q_{k-t-1}\left(u_{j}^{k-t}\right) g^{t}\left(u_{j}^{k-t}-2\right) .
\end{aligned}
$$

\footnotetext{
6) Задача о динамике полюсов эллиптических решений в первой постановке приводит к спиновым обобщениям систем Калоджеро-Мозера и Рейсенарса $[52,51]$. В нашем случае дело дополнительно осложняется открытыми граничными условиями типа Лиувилля, поэтому возможность буквального перенесения методов упомянутых работ на эту ситуацию пока не ясна.
} 
Разделив первое из этих соотношений на второе и подставив третье, можно исключить $g$ и получить систему уравнений на нули эллиптических полиномов $Q_{t}(u)$ :

$$
\frac{Q_{t-1}\left(u_{j}^{t}+2\right) Q_{t}\left(u_{j}^{t}-2\right) Q_{t+1}\left(u_{j}^{t}\right)}{Q_{t-1}\left(u_{j}^{t}\right) Q_{t}\left(u_{j}^{t}+2\right) Q_{t+1}\left(u_{j}^{t}-2\right)}=-1
$$

или в более подробной записи

$$
\prod_{l=1}^{M_{t-1}} \frac{\theta_{1}\left(\eta\left(u_{j}^{t}-u_{l}^{t-1}+2\right)\right)}{\theta_{1}\left(\eta\left(u_{j}^{t}-u_{l}^{t-1}\right)\right)} \prod_{l=1}^{M_{t}} \frac{\theta_{1}\left(\eta\left(u_{j}^{t}-u_{l}^{t}-2\right)\right)}{\theta_{1}\left(\eta\left(u_{j}^{t}-u_{l}^{t}+2\right)\right)} \prod_{l=1}^{M_{t+1}} \frac{\theta_{1}\left(\eta\left(u_{j}^{t}-u_{l}^{t+1}\right)\right)}{\theta_{1}\left(\eta\left(u_{j}^{t}-u_{l}^{t+1}-2\right)\right)}=-1 .
$$

Вместе с "граничными условиями"

$$
Q_{0}(u)=1, \quad Q_{k}(u)=\phi(u)
$$

эта система $M_{1}+M_{2}+\cdots+M_{k-1}$ уравнений эквивалентна уравнениям иерархического анзаца Бете для $A_{k-1}$-моделей с $R$-матрицей Белавина. Такие же уравнения получаются для функций на правом краю диаграммы (4.22). Ниже функции $Q_{t}$ будут отождествлены с теми, которые возникают при стандартном решении задачи анзацем Бете. Тем самым уравнения иерархии "вложенных" анзацев Бете можно понимать как "уравнения движения" для нулей эллиптического полинома $Q_{t}(u)$ в дискретном времени $t$ (которое имеет смысл "уровня" анзаца и пробегает вершины диаграммы Дынкина).

Отметим, что такое происхождение уравнений (4.24) позволяет считать их дискретным во времени аналогом уравнений движения эллиптической модели Рейсенарса. В непрерывном пределе по $t$ (при $M_{t}=M=$ const) уравнения (4.24) переходят в уравнения движения $M$-частичной модели Рейсенарса с эллиптическим потенциалом. Последующий предел $\eta \rightarrow 0$ c сохранением конечных значений $\eta u_{j}=x_{j}$ дает хорошо известные уравнения движения системы частиц Калоджеро-Мозера с эллиптическим потенциалом.

Системы частиц в дискретном времени имеют более сложные свойства, чем их непрерывные аналоги. В частности, число частиц в системе может зависеть от дискретного времени. В непрерывном времени это возможно только для решений с очень сингулярным поведением, когда частишы могут уходить на бесконечность или падать друг на друга за конечный отрезок времени. Именно этот случай реализуется для решений уравнений (4.24), соответствующих собственным состояниям гамильтониана квантовой модели, поскольку число возбуждений на $t$-м уровне иерархического анзаца Бете во всех известных примерах зависит от $t$. Это означает, что число "частиш" в дискретной модели Рейсенарса в этом случае не сохраняется. Тем не менее числа $M_{t}$ не могут быть произвольными. Для $A_{k-1}$-моделей с эллиптической $R$-матрицей в случае обшего положения (т.е. когда $\eta$ не является точкой конечного порядка на эллиптической кривой, заданной парой периодов 1 и $\tau) M_{t}=(N / k) t$, где $N$ - число узлов решетки, равное степени эллиптического полинома $\phi(u)$. В тригонометрическом и рациональном случаях 
условия на $M_{t}$ становятся менее ограничительными, но все равно эти числа не могут быть одинаковыми.

Тот факт, что степень эллиптического полинома $Q_{t}(u)$ равна $M_{t}=(N / k) t$ и $N$ должно делиться на $k$, следует прямо из уравнений (4.24). Действительно, из того, что $Q_{t}(u)$ - эллиптический полином, следует, что если $u_{j}^{t}-$ корень $Q_{t}(u)$, т.е. $Q_{t}\left(u_{j}^{t}\right)=0$, то $u_{j}^{t}+n_{1}+n_{2} \tau$ для всех целых $n_{1}, n_{2}-$ тоже его корни. Вспомнив правила преобразования $\theta$-функций при сдвиге на периоды, легко увидеть, что это возможно, если и только если выполняется соотношение

$$
M_{t+1}+M_{t-1}=2 M_{t}
$$

которое имеет единственное решение $M_{t}=(N / k) t$, удовлетворяюшее нужным граничным условиям. Это, в частности, означает, что иерархический анзац Бете применим к эллиптическим $A_{k-1}$-моделям, только если $N$ кратно $k$.

Таким образом, мы видим, что число частиц ведет себя подобно координате центра масс замкнутой системы - меняется линейно по времени. Соответствующий "импульс", т.е. прирашение числа частиц за шаг дискретного времени, сохраняется. Было бы интересно найти другие законы сохранения для динамической системы (4.25).

В тригонометрическом и рациональном случаях условия на степень $Q_{t}$ менее ограничительны, т.к. часть корней может уходить на бесконечность. Равенство в формуле для $M_{t}$ заменяется неравенством $M_{t} \leqslant(N / k) t$. Более детальный анализ [53] показывает, что должны выполняться еще и следующие неравенства: $2 M_{1} \leqslant M_{2}$, $2 M_{2} \leqslant M_{1}+M_{3}, \ldots, 2 M_{t} \leqslant M_{t-1}+M_{t+1}, \ldots, N=M_{k} \geqslant 2 M_{k-1}-M_{k-2}$.

Модель Рейсенарса в непрерывном времени была введена в [54] как релятивистское обобщение системы Калоджеро-Мозера. Уравнения движения системы Рейсенарса в дискретном времени впервые появились в работе [55], где они были выведены из некоторого анзаца для пары Лакса. Тесная связь с эллиптическими решениями уравнения Хироты и иерархическим анзацем Бете была обнаружена и объяснена в нашей работе [17].

\section{5. РАЗНОСТНЫЕ УРАВНЕНИЯ ДЛЯ ФУНКЦИЙ $Q_{t}$}

Функции $Q_{t}(u)$ удовлетворяют некоторым линейным разностным уравнениям. В принципе они могут быть получены из линейных задач $(4.17),(4.18)$ на уровнях $t=$ $1,2, \ldots, k$ путем исключения всех функций $T$ в $(4.22)$, кроме самых крайних. На примере $k=2$ это явным образом проделано в следуюшем разделе.

В общем случае принщип остается тот же - векторная задача первого порядка переписывается как скалярная задача более высокого порядка для одной из компонент, но с технической точки зрения эта процедура становится довольно сложной. Полное решение этой задачи подробно описано в [17]. Здесь мы приведем эти результаты и схему их получения в несколько упрошенном виде. Наш метод заключается в использовании обшего решения билинейных соотношений, которое зависит от свободных функциональных параметров. Оно представляет и самостоятельный интерес. 


\section{1. Общее решение билинейных соотношений}

Здесь будет предъявлено общее решение системы функциональных уравнений, состоящей из уравнений Хироты (3.1) на каждом уровне $t=0,1, \ldots$

$$
T_{s, t}^{a}(u+1) T_{s, t}^{a}(u-1)-T_{s+1, t}^{a}(u) T_{s-1, t}^{a}(u)=T_{s, t}^{a+1}(u) T_{s, t}^{a-1}(u)
$$

и уравнений $(4.17),(4.18)$

$$
\begin{aligned}
& T_{s+1, t+1}^{a+1}(u) T_{s, t}^{a}(u)-T_{s, t+1}^{a+1}(u+1) T_{s+1, t}^{a}(u-1)=T_{s, t+1}^{a}(u) T_{s+1, t}^{a+1}(u), \\
& T_{s+1, t+1}^{a}(u+1) T_{s, t}^{a}(u)-T_{s, t+1}^{a}(u) T_{s+1, t}^{a}(u+1)=T_{s, t+1}^{a+1}(u+1) T_{s+1, t}^{a-1}(u),
\end{aligned}
$$

связьвающих два соседних уровня. Приведем здесь еше три уравнения, которые можно получить из (5.1)-(5.3) алгебраическими преобразованиями:

$$
\begin{gathered}
T_{s, t+1}^{a}(u+1) T_{s, t}^{a}(u-1)-T_{s-1, t+1}^{a}(u) T_{s+1, t}^{a}(u)=T_{s, t+1}^{a+1}(u) T_{s, t}^{a-1}(u), \\
T_{s, t+1}^{a}(u+1) T_{s, t}^{a+1}(u)+T_{s-1, t+1}^{a+1}(u+1) T_{s+1, t}^{a}(u)=T_{s, t+1}^{a+1}(u) T_{s, t}^{a}(u+1), \\
T_{s, t+1}^{a}(u-1) T_{s, t}^{a}(u+1)-T_{s+1, t+1}^{a}(u) T_{s-1, t}^{a}(u)= \\
\quad=T_{s, t+1}^{a-1}(u) T_{s, t}^{a+1}(u)-T_{s-1, t+1}^{a+1}(u+1) T_{s+1, t}^{a-1}(u-1) .
\end{gathered}
$$

Граничные условия имеют вид (4.14) с дополнительным требованием, чтобы $T_{s, t}^{0}(u)$ и $T_{s, t}^{t}(u)$ при всех $t \geqslant 0$ зависели только от $u-s$ и $u+s$, соответственно, чего всегда можно добиться калибровочным преобразованием. Для определенности положим $T_{s, t}^{a}(u)=0$ при $t<0$ и $T_{s, 0}^{0}(u)=1$. Иными словами, мы хотим найти решение для всего массива (4.22) одновременно. При этом постановка задачи несколько меняется. Во-первых, функции $Q_{t}$ и $\bar{Q}_{t}$ на краях массива пока не считаются связанными соотношением (4.16). Во-вторых, мы не фиксируем уровень $k$ и функции $Q_{k}(u), \bar{Q}_{k}(u)$, а строим решение, двигаясь по массиву “в обратном направлении" - сверху вниз. В-третьих, от функций $Q_{t}, \bar{Q}_{t}$ мы перейдем к другому набору функциональных параметров, нули которых даже в эллиптических решениях не будут связаны уравнениями Бете. На каждом шаге (т.е. при переходе от $t$ к $t+1)$ добавляются два таких параметра.

Зададим два набора функций $q_{i}(u), \bar{q}_{i}(u), i=1,2, \ldots$, которые пока считаются произвольными и не зависимыми друг от друга. Они будут играть роль функциональных параметров в обшем решении. Единственное предположение - линейная независимость функций из каждого набора.

Теорема 5.1. Общее решение билинейных соотношений (5.1)-(5.3) с граничным условием (4.14) имеет вид

$$
T_{s, t}^{a}(u)=(-1)^{a(t-1)}\left|\begin{array}{ccc}
q_{t}(u-s-a) & \cdots & q_{1}(u-s-a) \\
q_{t}(u-s-a-2) & \cdots & q_{1}(u-s-a-2) \\
\ldots & \cdots & \ldots \\
q_{t}(u-s-2 t+a+2) & \cdots & q_{1}(u-s-2 t+a+2) \\
\bar{q}_{t}(u+s+a-1) & \cdots & \bar{q}_{1}(u+s+a-1) \\
\bar{q}_{t}(u+s+a-3) & \cdots & \bar{q}_{1}(u+s+a-3) \\
\cdots & \cdots & \cdots \\
\bar{q}_{t}(u+s-a+1) & \cdots & \bar{q}_{1}(u+s-a+1)
\end{array}\right| .
$$


ДокАЗАТЕЛЬСТВо заключается в применении тождества

$$
D\left[i_{1}, i_{3}\right] D\left[i_{2}, i_{4}\right]-D\left[i_{1}, i_{4}\right] D\left[i_{2}, i_{3}\right]=D\left[i_{1}, i_{2}\right] D\left[i_{3}, i_{4}\right]
$$

справедливого для миноров $D[i, j]$ произвольной прямоугольной $(n+2) \times n$-матрицы, получающихся вычеркиванием из нее двух строк с номерами $i, j, i<j$. Это тождествопростейший пример соотношений Плюккера (подробности см. в приложении).

Матрица в (5.7) имеет размер $t \times t$. Первые $t-a$ строк содержат функции $q_{i}(u-s)$, причем при сдвиге на строчку вниз их аргумент сдвигается на -2 , последние $a$ строк содержат $\bar{q}_{i}(u+s)$, аргумент которых в каждой следующей строчке опять сдвигается на -2. Не удивительно, что детерминантная формула (5.7) по своей структуре напоминает решение Лезнова-Савельева $[56,57]$ для двумеризованной цепочки Тоды с открытыми концами в непрерывном времени, дискретной версией которого она является.

На границах массива имеем

$$
\begin{aligned}
& T_{s, t}^{0}(u)=Q_{t}(u-s)=\operatorname{det}_{1 \leqslant i, j \leqslant t} q_{t+1-j}(u-s-2 i+2), \\
& T_{s, t}^{t}(u)=\overline{Q_{t}}(u+s)=\operatorname{det}_{1 \leqslant i, j \leqslant t} \bar{q}_{t+1-j}(u+s+t-2 i+1) .
\end{aligned}
$$

В частности, $q_{1}(u)=Q_{1}(u), \bar{q}_{1}(u)=\bar{Q}_{1}(u)$. Эти формулы дают параметризацию функций $Q_{t}, \bar{Q}_{t}$ через независимые функциональные параметры $q_{i}, \bar{q}_{i}$.

В дальнейшем для нас важно, что функциональные параметры $q_{i}(u)$ остаются независимыми и для интересуюшего нас класса решений, в которых функция $T_{s, t}^{a}(u)$ должна быть эллиптическим полиномом от $u$. Именно, если положить $\bar{q}_{i}(u)=q_{i}(u+1)$ и выбрать $q_{i}(u)$ в виде (произвольных!) эллиптических полиномов одной и той же степени для всех $i=1,2, \ldots$, мы получаем решение, удовлетворяющее всем свойствам, которые требуются от $T$-матриц квантовой задачи.

\section{2. Обобщенные соотношения Бакстера}

Обобшенными соотношениями Бакстера мы называем разностные уравнения, которым удовлетворяют функции $Q_{t}$. Для выяснения их структуры можно воспользоваться общим решением из предыдущего пункта 5.1. Важное техническое преимушество, которое дает этот способ, - это то, что построенное решение зависит от свободныц функциональных параметров. В терминах нашего обшего решения смысл следуюших ниже громоздких формул будет абсолютно прозрачен. Одновременно станет ясен смысл функциональных параметров $q_{i}, \bar{q}_{i}$.

Объясним способ вывода обобщенных соотношений Бакстера на примере $Q_{1}(u)=$ $q_{1}(u)$. Фиксируем какой-нибудь уровень $k \geqslant 2$ и положим $T_{s, k}^{a}(u)=T_{s}^{a}(u)$, как и раньше. Рассмотрим числовую $(k+1) \times(k+1)$-матрицу

$$
\mathbf{T}_{i j} \equiv T_{s+i+j}^{k-1}(u+s-i+j), \quad i, j=1,2, \ldots, k+1
$$


Разложение детерминанта (5.7) по первой строке имеет вид

$$
\mathbf{T}_{i j}=\sum_{l=1}^{k} q_{l}(u-k+1-2 i) \mathbf{M}_{l}(u+2 s+2 j),
$$

где $\mathbf{M}_{l}(u+2 s+2 j)$ - миноры матрицы (5.7). Отсюда ясно, что $\mathbf{T}_{i j}$ - матрица ранга не более $k$. Следовательно, $\operatorname{det} \mathbf{T}_{i j}=0$. Очевидно также, что детерминанты матриш, полученных из $\mathbf{T}_{i j}$ заменой любого ее столбца на столбец $q_{t}(u-k+1-2 i)$, составленный из значений любой из функций $q_{t}(u) \mathrm{c} 1 \leqslant t \leqslant k$, тоже равны нулю. Тем самым для любого $t=1,2, \ldots, k$ получаем тождества

$$
\left|\begin{array}{ccccc}
T_{s}^{k-1}(u) & T_{s+1}^{k-1}(u+1) & \ldots & T_{s+k-1}^{k-1}(u+k-1) & q_{t}(u-s-k+1) \\
T_{s+1}^{k-1}(u-1) & T_{s+2}^{k-1}(u) & \ldots & T_{s+k}^{k-1}(u+k-2) & q_{t}(u-s-k-1) \\
\ldots & \ldots & \ldots & \ldots & \ldots \\
T_{s+k}^{k-1}(u-k) & T_{s+k+1}^{k-1}(u-k+1) & \ldots & T_{s+2 k-1}^{k-1}(u-1) & q_{t}(u-s-3 k+1)
\end{array}\right|=0 .
$$

Разложив детерминант по последнему столбцу, мы можем записать их в виде

$$
\sum_{i=0}^{k} U_{i}(u, s) q_{t}(u-2 i)=0
$$

Нормируем коэффициенты так, чтобы $U_{0}(u, s)=1$ (т.е. поделим все на соответствуюший минор матрицы T). Из (5.12) следует, что в такой нормировке $U_{i}(u, s)$ не зависят om s. Действительно, перепишем (5.12) как систему $k$ линейных уравнений на $k$ неизвестных $U_{i}(u, s)$ :

$$
\sum_{i=1}^{k} q_{t}(u-2 i) U_{i}(u, s)=-q_{t}(u), \quad t=1,2, \ldots, k
$$

Ее детерминант отличен от нуля в силу линейной независимости функций $q_{i}$. Следовательно, система имеет единственное решение. Поскольку ее коэффициенты не зависят от $s$, то же верно и для $U_{i}$.

Итак, приходим к выводу, что функция $Q_{1}(u)=q_{1}(u)$ является одним из решений разностного уравнения

$$
\left|\begin{array}{ccccc}
T_{s}^{k-1}(u) & T_{s+1}^{k-1}(u+1) & \ldots & T_{s+k-1}^{k-1}(u+k-1) & Q_{1}(u-s-k+1) \\
T_{s+1}^{k-1}(u-1) & T_{s+2}^{k-1}(u) & \ldots & T_{s+k}^{k-1}(u+k-2) & Q_{1}(u-s-k-1) \\
\ldots & \ldots & \ldots & \ldots & \ldots \\
T_{s+k}^{k-1}(u-k) & T_{s+k+1}^{k-1}(u-k+1) & \ldots & T_{s+2 k-1}^{k-1}(u-1) & Q_{1}(u-s-3 k+1)
\end{array}\right|=0
$$

коэффициенты которого после обшего сдвига на $u \rightarrow u+s$ и вынесения общего множителя перестают зависеть от $s$. Остальными линейно независимыми решениями этого 
уравнения являются функции $q_{2}, q_{3}, \ldots, q_{k}$. Фактически мы написали уравнение по известному набору его решений - прием, хорошо известный в теории цепочки Тоды с открытыми концами в непрерывном времени (см., например, [57]), где (5.13) преврашается в дифференциальное уравнение $k$-го порядка. Аналогичным образом можно получить уравнения

$$
\begin{gathered}
\left|\begin{array}{ccccc}
T_{s}^{1}(u) & T_{s-1}^{1}(u+1) & \ldots & T_{s-k+1}^{1}(u+k-1) & \bar{Q}_{1}(u+s) \\
T_{s-1}^{1}(u-1) & T_{s-2}^{1}(u) & \ldots & T_{s-k}^{1}(u+k-2) & \bar{Q}_{1}(u+s-2) \\
\ldots & \ldots & \ldots & \ldots & \ldots \\
T_{s-k}^{1}(u-k) & T_{s-k-1}^{1}(u-k+1) & \ldots & T_{s-2 k+1}^{1}(u-1) & \bar{Q}_{1}(u+s-2 k)
\end{array}\right|=0, \\
\left|\begin{array}{ccccc}
T_{s}^{1}(u) & T_{s+1}^{1}(u+1) & \ldots & T_{s+k-1}^{1}(u+k-1) & Q_{k-1}(u-s-1) \\
T_{s+1}^{1}(u-1) & T_{s+2}^{1}(u) & \ldots & T_{s+k}^{1}(u+k-2) & Q_{k-1}(u-s-3) \\
\ldots & \ldots & \ldots & \ldots & \ldots \\
T_{s+k}^{1}(u-k) & T_{s+k+1}^{1}(u-k+1) & \ldots & T_{s+2 k-1}^{1}(u-1) & Q_{k-1}(u-s-2 k-1)
\end{array}\right|=0, \\
\begin{array}{ccccc}
T_{s}^{k-1}(u) & T_{s-1}^{k-1}(u+1) & \ldots & T_{s-k+1}^{k-1}(u+k-1) & \bar{Q}_{k-1}(u+s) \\
T_{s-1}^{k-1}(u-1) & T_{s-2}^{k-1}(u) & \ldots & T_{s-k}^{k-1}(u+k-2) & \bar{Q}_{k-1}(u+s-2) \\
\ldots & \ldots & \ldots & \ldots & \ldots \\
T_{s-k}^{k-1}(u-k) & T_{s-k-1}^{k-1}(u-k+1) & \ldots & T_{s-2 k+1}^{k-1}(u-1) & \bar{Q}_{k-1}(u+s-2 k)
\end{array} \mid=0
\end{gathered}
$$

для функций $\bar{Q}_{1}, Q_{k-1}, \bar{Q}_{k-1}$, соответственно. После общего сдвига $u \rightarrow u \pm s$ и вынесения общего множителя коэффициенты этих уравнений перестают зависеть от $s$. Эти уравнения можно рассматривать как обобщения $T$ - $Q$-соотношения Бакстера.

Уравнения для $Q_{t}$ с $2 \leqslant t \leqslant k-2$ имеют более сложный вид. Они получаются аналогичной процедурой из детерминантной формулы (5.7) с помошью разложения Лапласа. В обшем случае $Q_{t}(u), \bar{Q}_{t}(u)$ удовлетворяют разностным уравнениям порядка $C_{k}^{a}+1$, где $C_{k}^{a}$ - биномиальный коэффициент. Мы не будем их здесь обсуждать.

Подстановка условий (3.6), (3.9), (4.16), специфичных для решений типа анзаца Бете, приводит к значительным упрошениям коэффишиентов выписанных уравнений. Кроме того, уравнения для $Q_{t}$ и $\bar{Q}_{t}$ становятся одинаковыми. Детерминанты в $(5.13),(5.14)$ дают при этом уравнение

$$
\sum_{a=0}^{k}(-1)^{a} T_{1}^{a}(u-a+1) Q_{1}(u-2 a+2)=0,
$$

$\mathrm{a}(5.15),(5.16)-$

$$
\sum_{a=0}^{k}(-1)^{a} \frac{T_{1}^{a}(u+a+1)}{\phi(u+2 a+2)} \frac{Q_{k-1}(u+2 a)}{\phi(u+2 a)}=0 .
$$

Напомним, что мы пришли к этим соотношениям, используя только уравнение Хироты с нужными граничными условиями. 


\section{3. Формулы факторизации}

Чтобы установить соответствие со стандартным иерархическим анзацем Бете, надо отождествить наши $Q_{t}$ с теми, которые фигурируют в решении, полученном стандартным методом. Один из способов это сделать - факторизовать разностные операторы в (5.17) и (5.18) в терминах $Q_{t}(u)$. Одновременно это даст формулы, позволяюшие выразить $T_{s}^{a}(u)$ через $Q_{t}(u)$.

ПРЕДЛОЖЕНИЕ 5.1. Для разностного оператора в левой части (5.17) справедлива следующая формула факторизации:

$$
\begin{array}{r}
\sum_{a=0}^{k}(-1)^{a-k} \frac{T_{1}^{a}(u-a+1)}{\phi(u+2)} e^{-2 a \partial_{u}}=\left(e^{-2 \partial_{u}}-\frac{Q_{k}(u) Q_{k-1}(u+2)}{Q_{k}(u+2) Q_{k-1}(u)}\right) \times \cdots \\
\cdots \times\left(e^{-2 \partial_{u}}-\frac{Q_{2}(u) Q_{1}(u+2)}{Q_{2}(u+2) Q_{1}(u)}\right)\left(e^{-2 \partial_{u}}-\frac{Q_{1}(u)}{Q_{1}(u+2)}\right) .
\end{array}
$$

ДоКАЗАТЕЛЬСТВо. Поскольку старшие коэффициенты операторов в обеих частях равенства одинаковы, достаточно убедиться, что оператор в правой части обращает в нуль все функции $q_{1}(u+2), q_{2}(u+2), \ldots, q_{k}(u+2)$. Для $q_{1}(u+2)$ это очевидно. Далее, ясно, что $T$-функции на уровне $k$, даваемые формулой (5.7), не меняются при одновременном изменении нумерации функций $q_{t}, \bar{q}_{t} \mathrm{c} 1 \leqslant t \leqslant k$ (с точностью до общего знака). В то же время величины $Q_{t}$ для $1 \leqslant t \leqslant k-1$, вообще говоря, зависят от этой нумерации.

Рассмотрим элементарную транспозицию $\pi_{t}(t)=t+1, \pi_{t}(t+1)=t$ и $\pi_{t}(i)=i$ при $i \neq t, t+1$. Пусть $\check{q}_{i} \equiv q_{\pi_{t}(i)}$ - переставленная последовательность функциональных параметров. (Здесь и ниже подразумевается, что $\bar{q}_{t}$ переставляются таким же образом.) Через $\check{Q}_{i}$ обозначим соответствующую последовательность $Q$-фоннкций, построенных по формуле (5.9). Ясно, что $\check{Q}_{i}=Q_{i}$ при $1 \leqslant i \leqslant t-1, \check{Q}_{i}=-Q_{i}$ при $t+1 \leqslant$ $i \leqslant k$, а $\check{Q}_{t}$ дается детерминантом (5.9), первый столбец которого состоит из функций $q_{t+1}$ вместо $q_{t}$. В приложении показано, что из детерминантного тождества (5.8) вытекает билинейное соотношение

$$
\check{Q}_{t}(u+2) Q_{t}(u)-Q_{t}(u+2) \check{Q}_{t}(u)=Q_{t+1}(u+2) Q_{t-1}(u) .
$$

Его непосредственным следствием является формула

$$
\begin{aligned}
& \left(e^{-2 \partial_{u}}-\frac{Q_{t+1}(u) Q_{t}(u+2)}{Q_{t+1}(u+2) Q_{t}(u)}\right)\left(e^{-2 \partial_{u}}-\frac{Q_{t}(u) Q_{t-1}(u+2)}{Q_{t}(u+2) Q_{t-1}(u)}\right)= \\
& =\left(e^{-2 \partial_{u}}-\frac{Q_{t+1}(u) \check{Q}_{t}(u+2)}{Q_{t+1}(u+2) \check{Q}_{t}(u)}\right)\left(e^{-2 \partial_{u}}-\frac{\check{Q}_{t}(u) Q_{t-1}(u+2)}{\check{Q}_{t}(u+2) Q_{t-1}(u)}\right)
\end{aligned}
$$

применяя которую для $\pi_{t}, \pi_{t+1}$ и т. д., можно переместить $q_{t+1}$ в самый правый операторный сомножитель, после чего обрашение в нуль функции $q_{t}(u+2)$ оператором (5.19) станет очевидным. 
Укажем другие полезные варианты формулы факторизации. Оператор, сопряженньй к (5.19), дает факторизацию разностного оператора в (5.18):

$$
\begin{aligned}
& \sum_{a=0}^{k}(-1)^{a-k} \frac{T_{1}^{a}(u+a+1)}{\phi(u+2 a+2)} e^{2 a \partial_{u}}=\left(e^{2 \partial_{u}}-\frac{Q_{1}(u)}{Q_{1}(u+2)}\right) \times \\
& \quad \times\left(e^{2 \partial_{u}}-\frac{Q_{2}(u) Q_{1}(u+2)}{Q_{2}(u+2) Q_{1}(u)}\right) \ldots\left(e^{2 \partial_{u}}-\frac{Q_{k}(u) Q_{k-1}(u+2)}{Q_{k}(u+2) Q_{k-1}(u)}\right) .
\end{aligned}
$$

Формулу (5.22) можно переписать в виде

$$
\begin{aligned}
& \sum_{a=0}^{k}(-1)^{a} \frac{T_{1}^{a}(u+a-1)}{\phi(u+2 a-2)} e^{2 a \partial_{u}}=\left(1-\frac{Q_{1}(u+2)}{Q_{1}(u)} e^{2 \partial_{u}}\right) \times \\
& \quad \times\left(1-\frac{Q_{2}(u+2) Q_{1}(u-2)}{Q_{2}(u) Q_{1}(u)} e^{2 \partial_{u}}\right) \ldots\left(1-\frac{Q_{k}(u+2) Q_{k-1}(u-2)}{Q_{k}(u) Q_{k-1}(u)} e^{2 \partial_{u}}\right) .
\end{aligned}
$$

Коэффициенты оператора, обратного к (5.23), дают $T_{s}^{1}(u)$ :

$$
\begin{gathered}
\sum_{s=0}^{\infty} \frac{T_{s}^{1}(u+s-1)}{\phi(u)} e^{2 s \partial_{u}}=\left(1-\frac{Q_{k}(u+2) Q_{k-1}(u-2)}{Q_{k}(u) Q_{k-1}(u)} e^{2 \partial_{u}}\right)^{-1} \times \cdots \\
\cdots \times\left(1-\frac{Q_{2}(u+2) Q_{1}(u-2)}{Q_{2}(u) Q_{1}(u)} e^{2 \partial_{u}}\right)^{-1}\left(1-\frac{Q_{1}(u+2)}{Q_{1}(u)} e^{2 \partial_{u}}\right)^{-1}
\end{gathered}
$$

Эти формулы позволяют выразить $T_{1}^{a}(u)$ и $T_{s}^{1}(u)$ в терминах эллиптических полиномов $Q_{t}$, корни которых определяются из уравнений Бете. При этом условии $T_{1}^{a}(u)$ не содержит полюсных членов. Наконец, $T_{s}^{a}(u)$ при $a, s>1$ могут быть найдены из детерминантных формул $(2.31),(2.32)$.

Левая часть соотношения (5.23) известна как производяшая функция для $T_{1}^{a}(u)$. Эти формулы для производяшей функции совпадают с известными в литературе (например, $[34,58,59])$. Обычно их трактуют как некоммутативные аналоги производящих функций для симметрических полиномов от многих переменных. В следуюшем разделе на примере $A_{1}$-моделей будет предложена другая их интерпретация.

\section{6. $A_{1}-$ МОДЕЛИ}

Формулы предыдушего раздела полезно проиллюстрировать на примере $A_{1}$-моделей. Мы также обсуждаем специфику случая высших представлений в квантовом пространстве и предлагаем новую интерпретацию $T$ - $Q$-соотношения Бакстера как разностного уравнения для функции Бейкера-Ахиезера. 


\section{1. Общее решение дискретного уравнения Лиувилля}

Соотношение (5.7) для $A_{1}$-моделей преврашается в дискретное уравнение Лиувилля в билинейной форме, переписанное в “лабораторных” переменных:

$$
T_{s}(u+1) T_{s}(u-1)-T_{s+1}(u) T_{s-1}(u)=\phi(u-s) \bar{\phi}(u+s) .
$$

Здесь и далее мы упростили обозначения, положив $T_{s}(u) \equiv T_{s}^{1}(u)$. Функции $\phi, \bar{\phi}$, вообше говоря, независимы. В решениях, воспроизводящих результаты анзаца Бете, надо накладывать связь $\bar{\phi}(u)=\phi(u+2)$.

Линейные задачи дают всего два нетривиальных уравнения:

$$
\begin{aligned}
T_{s+1}(u) Q(u-s)-T_{s}(u+1) Q(u-s-2) & =\phi(u-s) \bar{Q}(u+s+1), \\
T_{s+1}(u) \bar{Q}(u+s-1)-T_{s}(u-1) \bar{Q}(u+s+1) & =\bar{\phi}(u+s) Q(u-s-2) .
\end{aligned}
$$

Здесь $Q(u) \equiv Q_{1}(u), \phi(u)=Q_{2}(u)$ и т.д. Отметим, что если $Q$ и $\bar{Q}$ удовлетворяют уравнениям (6.2), (6.3), то функция

$$
\widetilde{T}_{s}(u)=T_{s}(u)+\alpha Q(u-s-1) \bar{Q}(u+s)
$$

с произвольной константой $\alpha$ удовлетворяет (6.1).

Поделив обе части (6.2) на $\phi(u-s)$ и воспользовавшись тем, что правая часть получившегося соотношения не зависит от $u-s$, а затем проделав аналогичную процедуру со вторым уравнением, придем к соотношениям

$$
\begin{aligned}
& \phi(u) Q(u+2)+\phi(u+2) Q(u-2)=A(u) Q(u) \\
& \bar{\phi}(u) \bar{Q}(u-3)+\bar{\phi}(u-2) \bar{Q}(u+1)=\bar{A}(u) \bar{Q}(u-1)
\end{aligned}
$$

и условию связи

$$
T_{1}(u) Q(u)-T_{0}(u+1) Q(u-2)=\phi(u) \bar{Q}(u+1),
$$

которое следует из (6.2) при $s=0$. В этих уравнениях функции

$$
\begin{aligned}
A(u) & =\frac{\phi(u+2) T_{s+1}(u+s)+\phi(u) T_{s-1}(u+s+2)}{T_{s}(u+s+1)}, \\
\bar{A}(u) & =\frac{\bar{\phi}(u-2) T_{s+1}(u-s)+\bar{\phi}(u) T_{s-1}(u-s-2)}{T_{s}(u-s-1)}
\end{aligned}
$$

не зависят от $s$ в силу (6.1).

Функция

$$
Y_{s}(u)=\frac{T_{s+1}(u) T_{s-1}(u)}{\phi(u-s) \phi(u+s+2)}
$$

удовлетворяет дискретному уравнению Лиувилля

$$
Y_{s}(u-1) Y_{s}(u+1)=\left(Y_{s+1}(u)+1\right)\left(Y_{s-1}(u)+1\right) .
$$


Общее решение строится совершенно аналогично непрерывной теории (см., например, работы [60-62] и приведенные там ссылки). Пусть $q_{1}, q_{2}$ (соответственно $\left.\bar{q}_{1}, \bar{q}_{2}\right)$ - два линейно независимых решения уравнения (6.4) (соответственно (6.5)), причем функции $A(u), \bar{A}(u)$ определяются из начальных данных. Нормируем их так, чтобы выполнялись соотношения для вронскианов

$$
\begin{aligned}
& \left|\begin{array}{cc}
q_{2}(u) & q_{1}(u) \\
q_{2}(u-2) & q_{1}(u-2)
\end{array}\right|=\phi(u), \\
& \left|\begin{array}{cc}
\bar{q}_{2}(u) & \bar{q}_{1}(u) \\
\bar{q}_{2}(u-2) & \bar{q}_{1}(u-2)
\end{array}\right|=\bar{\phi}(u-1),
\end{aligned}
$$

а также условие связи

$$
T_{1}(u) q_{2}(u)-T_{0}(u+1) q_{2}(u-2)=\phi(u) \bar{q}_{2}(u+1)
$$

вытекающее из (6.6). Тогда обшее решение уравнения (6.1) имеет вид

$$
T_{s}(u)=\left|\begin{array}{cc}
q_{1}(u-s-1) & q_{2}(u-s-1) \\
\bar{q}_{1}(u+s) & \bar{q}_{2}(u+s)
\end{array}\right|
$$

(ср. с (5.7)). Как и в непрерывном случае, это выражение инвариантно по отношению к выбору пары линейно независимых решений с данными вронскианами. Переход к другой паре решений описывается преобразованием из группы $S L(2)$. Благодаря (6.6) и (6.11) $\bar{q}_{1}$ и $\bar{q}_{2}$ преобразуются так же, как $q_{1}, q_{2}$, и инвариантность величины $(6.12)$ очевидна.

\section{2. Различные формы соотношения Бакстера}

Наложение связей $(4.16) \bar{\phi}(u)=\phi(u+2)$ и т.д. немедленно приводит к "начальному условию"

$$
T_{-1}(u)=0
$$

специфичному для квантовых $T$-матриц. Обратно, это условие вместе с требованием эллиптичности налагает связь между $\bar{Q}_{t}$ и $Q_{t}$ вида (4.16). Подставив это условие в $(6.7),(6.8)$, получим

$$
A(u)=\bar{A}(u)=T_{1}(u)
$$

Следовательно, весь набор уравнений сводится к соотношениям

$$
\begin{gathered}
T_{s}(u+1) T_{1}(u-s)=\phi(u-s+2) T_{s+1}(u)+\phi(u-s) T_{s-1}(u+2), \\
T_{s}(u-1) T_{1}(u+s)=\phi(u+s) T_{s+1}(u)+\phi(u+s+2) T_{s-1}(u-2), \\
\phi(u) Q(u+2)+\phi(u+2) Q(u-2)=T_{1}(u) Q(u) .
\end{gathered}
$$


Первые два - это ранние примеры “правил слияния", найденные в [36, 63], а (6.16) знаменитое $T$-Q-соотношение Бакстера $[64,65]$. Укажем еше две его эквивалентные формы:

$$
\begin{gathered}
\left|\begin{array}{ccc}
T_{s}^{1}(u) & T_{s+1}^{1}(u+1) & Q(u-s-1) \\
T_{s+1}^{1}(u-1) & T_{s+2}^{1}(u) & Q(u-s-3) \\
T_{s+2}^{1}(u-2) & T_{s+3}^{1}(u-1) & Q(u-s-5)
\end{array}\right|=0, \\
\left(e^{-2 \partial_{u}}-\frac{\phi(u-2) q_{1}(u)}{\phi(u) q_{1}(u-2)}\right)\left(e^{-2 \partial_{u}}-\frac{q_{1}(u-2)}{q_{1}(u)}\right) Q(u)=0,
\end{gathered}
$$

которые хорошо иллюстрируют общие формулы предыдушего раздела. Последнее равенство надо понимать как разностное уравнение на функцию $Q(u)$, имеюшее два решения, одно из которых совпадает с $q_{1}(u)$. Наконец, формула (6.12) преврашается в

$$
T_{s}(u)=\left|\begin{array}{ll}
q_{1}(u-s-1) & q_{2}(u-s-1) \\
q_{1}(u+s+1) & q_{2}(u+s+1)
\end{array}\right| .
$$

Уравнения Бете следуют, например, из (6.16) и имеют стандартный вид

$$
\frac{\phi\left(u_{j}+2\right)}{\phi\left(u_{j}\right)}=-\frac{Q\left(u_{j}+2\right)}{Q\left(u_{j}-2\right)},
$$

где $u_{j}$ - корни полинома $Q(u)$. Их интерпретация как динамической системы в дискретном времени в данном случае не очень содержательна, т.к. дискретное время пробегает всего три значения $t=0,1,2$, причем $Q_{0}(u)=1, Q_{1}(u)=Q(u), Q_{2}(u)=\phi(u)$.

\section{3. Высшие спины в квантовом пространстве}

Уравнение Хироты в минимальной нормировке (6.1), т.е.

$$
T_{s}(u+1) T_{s}(u-1)-T_{s+1}(u) T_{s-1}(u)=\phi(u-s) \phi(u+s+2),
$$

остается верным для любого квантового пространства. Однако, если нули $\phi(u)$ не находятся в обшем положении, а расположены специальным образом, $T_{s}(u)$ может содержать множитель, обший для всех полиномиальных решений. В этом и заключается специфика случая высших спинов в квантовом пространстве.

Пусть, например, $\phi(u)$ имеет вид

$$
\phi(u)=\prod_{p=1}^{2 \ell} \varphi(u+1+2 \ell-2 p),
$$

где $\varphi(u)$ - полином степени $N$ вида $(2.40)$ с корнями $y_{j}$ в общем положении. Такая функция $\phi(u)$ соответствует неоднородной спиновой цепочке на $N$ узлах со спином $\ell$ в каждом узле. Если $s<2 \ell$, из $T_{s}(u)$ можно вынести обший множитель

$$
\prod_{p=1}^{2 \ell-s} \varphi(u-s+2+2 \ell-2 p)
$$


т.е. величина

$$
\widetilde{T}_{s}(u)=\frac{T_{s}(u)}{\prod_{p=1}^{2 \ell-s} \varphi(u-s+2 \ell+2-2 p)}, \quad s \leqslant 2 \ell
$$

является полиномом степени $N s$. Переписав уравнение $(6.20)$ для $\widetilde{T}_{s}(u)$, получим

$$
\widetilde{T}_{s}(u+1) \widetilde{T}_{s}(u-1)-\widetilde{T}_{s+1}(u) \widetilde{T}_{s-1}(u)=\prod_{j=0}^{s-1} \varphi(u-s-2 \ell+1+2 j) \varphi(u-s+2 \ell+3+2 j)
$$

при $s \leqslant 2 \ell$ с начальным условием $\widetilde{T}_{0}(u)=1$. При $s>2 \ell$ имеем $\widetilde{T}_{s}(u)=T_{s}(u)$. Соотношение Бакстера (6.16) перепишется в виде

$$
\varphi(u+1-2 \ell) Q(u+2)+\varphi(u+1+2 \ell) Q(u-2)=\widetilde{T}_{1}(u) Q(u)
$$

\section{4. Соотношение Бакстера как разностное уравнение для функции Бейкера-Ахиезера}

Покажем, что соотношение Бакстера (6.16) можно проинтерпретировать как специальный случай разностных уравнений на функцию Бейкера-Ахиезера.

Здесь мы сушественно используем материал, изложенный в [29], и придерживаемся введенных там обозначений. Рассмотрим разностные операторы

$$
\mathcal{M}^{(a)}=e^{\partial_{x}}-\lambda_{a x} \frac{\tau^{a, b}(x) \tau^{a+1, b}(x+1)}{\tau^{a+1, b}(x) \tau^{a, b}(x+1)}, \quad \mathcal{M}^{(b)}=e^{\partial_{x}}-\lambda_{b x} \frac{\tau^{a, b}(x) \tau^{a, b+1}(x+1)}{\tau^{a, b+1}(x) \tau^{a, b}(x+1)}
$$

где $\tau^{a, b}(x)-\tau$-функция, зависящая в данном случае от трех дискретных переменных $a, b$ и $x$, а $\lambda_{a}, \lambda_{b}, \lambda_{x}-$ комплексные параметры; $\lambda_{a b} \equiv \lambda_{a}-\lambda_{b}$ и т.д. Уравнение Хироты является условием совместности линейных задач

$$
\mathcal{M}^{(a)} \psi^{a, b}(x ; z)=\psi^{a+1, b}(x ; z), \quad \mathcal{M}^{(b)} \psi^{a, b}(x ; z)=\psi^{a, b+1}(x ; z)
$$

для функции $\psi^{a, b}(x ; z)$, зависяшей от спектрального параметра $z$. Их решение, обладающее определенными свойствами, которые мы не будем здесь повторять, называется функцией Бейкера-Ахиезера. Из этих свойств для нас важны сейчас только следующие: в окрестности точки $z=\lambda_{a}$

$$
\psi^{a, b}(x ; z)=\left(z-\lambda_{a}\right)^{a}\left(\xi_{0}^{(a)}+O\left(z-\lambda_{a}\right)\right),
$$

а в окрестности точки $z=\lambda_{b}$

$$
\psi^{a, b}(x ; z)=\left(z-\lambda_{b}\right)^{b}\left(\xi_{0}^{(b)}+O\left(z-\lambda_{b}\right)\right),
$$

где $\xi_{0}^{(a)}, \xi_{0}^{(b)}$ - некоторые константы. В частности,

$$
\psi^{1,1}\left(u ; \lambda_{a}\right)=\psi^{1,1}\left(u ; \lambda_{b}\right)=0 .
$$


Рассмотрим теперь последовательное действие введенных разностных операторов:

$$
e^{\partial_{a}} \mathcal{M}^{(b)} e^{-\partial_{a}} \mathcal{M}^{(a)} \psi^{a, b}(x ; z)=\psi^{a+1, b+1}(x ; z)
$$

(перемножаемых так же, как и в одной из частей условия нулевой кривизны). Положив $a=b=0$, получаем, что функции

$$
\begin{aligned}
& f^{(1)}(x) \equiv \psi^{0,0}\left(x ; \lambda_{a}\right)=\left(\lambda_{a x}\right)^{x} \frac{\tau^{1,0}(x)}{\tau^{0,0}(x)} \\
& f^{(2)}(x) \equiv \psi^{0,0}\left(x ; \lambda_{b}\right)=\left(\lambda_{b x}\right)^{x} \frac{\tau^{0,1}(x)}{\tau^{0,0}(x)}
\end{aligned}
$$

являются двумя линейно независимыми решениями уравнения

$$
\left(e^{\partial_{x}}-\lambda_{b x} \frac{\tau^{1,0}(x) \tau^{1,1}(x+1)}{\tau^{1,1}(x) \tau^{1,0}(x+1)}\right)\left(e^{\partial_{x}}-\lambda_{a x} \frac{\tau^{0,0}(x) \tau^{1,0}(x+1)}{\tau^{1,0}(x) \tau^{0,0}(x+1)}\right) f(x)=0,
$$

которое после раскрытия скобок принимает вид

$$
\begin{aligned}
f(x & +1)+\lambda_{a x} \lambda_{b x} \frac{\tau^{1,1}(x) \tau^{0,0}(x-1)}{\tau^{0,0}(x) \tau^{1,1}(x-1)} f(x-1)= \\
& =\left[\lambda_{a x} \frac{\tau^{0,0}(x) \tau^{1,0}(x+1)}{\tau^{1,0}(x) \tau^{0,0}(x+1)}+\lambda_{b x} \frac{\tau^{1,1}(x) \tau^{1,0}(x-1)}{\tau^{1,0}(x) \tau^{1,1}(x-1)}\right] f(x) .
\end{aligned}
$$

Отождествление уравнения (6.28) с соотношением Бакстера достигается следуюшими подстановками:

$$
\begin{gathered}
f(x)=\left(\lambda_{a x} \lambda_{b x}\right)^{\frac{1}{2} x} \frac{Q(2 x)}{\tau^{0,0}(x)}, \\
\phi(2 x)=\tau^{0,0}(x) \tau^{1,1}(x-1), \\
T_{1}(2 x)=\frac{\left(\lambda_{a x} / \lambda_{b x}\right)^{\frac{1}{2}} \phi(2 x) \tau^{1,0}(x+1)+\left(\lambda_{a x} / \lambda_{b x}\right)^{-\frac{1}{2}} \phi(2 x+2) \tau^{1,0}(x-1)}{\tau^{1,0}(x)} .
\end{gathered}
$$

Уравнение (6.28) при этом примет форму (6.16) с $u=2 x$. Два его решения $q_{1}, q_{2}$ имеют вид

$$
\begin{aligned}
& q_{1}(2 x)=\left(\frac{\lambda_{a x}}{\lambda_{b x}}\right)^{\frac{1}{2} x} \tau^{1,0}(x), \\
& q_{2}(2 x)=\left(\frac{\lambda_{a x}}{\lambda_{b x}}\right)^{-\frac{1}{2} x} \tau^{0,1}(x) .
\end{aligned}
$$

Уравнение Хироты позволяет преобразовать (6.30) и (6.31) к виду

$$
\begin{aligned}
\phi(2 x) & =\frac{\left[\lambda_{b x} \tau^{0,1}(x) \tau^{1,0}(x-1)-\lambda_{a x} \tau^{1,0}(x) \tau^{0,1}(x-1)\right]}{\lambda_{b a}}, \\
T_{1}(u) & =\frac{\left(\lambda_{b x}\right)^{2} \tau^{1,0}(x-1) \tau^{0,1}(x+1)-\left(\lambda_{a x}\right)^{2} \tau^{1,0}(x+1) \tau^{0,1}(x-1)}{\lambda_{b a}\left(\lambda_{b x} \lambda_{a x}\right)^{\frac{1}{2}}},
\end{aligned}
$$


в котором мы узнаем детерминантные формулы (6.9) и (6.19). Подчеркнем, что мы получили их, используя только самые общие свойства функции Бейкера-Ахиезера.

Итак, разностное уравнение (6.28) формально отождествлено с $T$ - $Q$-соотношением Бакстера (в факторизованном виде это уравнение (6.27)). Чтобы отождествить их на содержательном уровне, надо обеспечить правильные аналитические свойства входяших в него функций. А именно, как видно из сравнения формул (4.23) и (6.29), функция Бейкера-Ахиезера должна быть двоякоблоховской ${ }^{7)}$ (см. [51]). Тем самым мы попадаем в ситуацию, хорошо изученную в работах $[48,52,51,49]$ об эллиптических решениях уравнений Кадомцева-Петвиашвили, цепочки Тоды и т.д. Из них следует, что все интересуюшие нас решения лежат в классе “конечнозонных" (алгеброгеометрических) и строятся по комплексным кривым конечного рода, которые являются накрытиями эллиптической кривой. Эти кривые реализуются как спектральные кривые оператора Лакса для системы Рейсенарса. Ниже мы называем их кривыми Рейсенарса.

Несколько слов о смысле полученных формул. На первый взгляд они отличаются от написанных в начале этого раздела только обозначениями. Тем не менее предложенный здесь способ их вывода, не апеллирующий к специфическим граничным условиям (3.5) лиувиллевского типа, погружает их в совершенно другой контекст. Теория конечнозонного интегрирования солитонных уравнений может дать конструктивный метод нахождения функций с нужными аналитическими свойствами, удовлетворяющих соотношению Бакстера. По некоторой кривой Рейсенарса можно построить сразу все три функции $\phi, Q$ и $T_{1}$, связанные соотношением Бакстера, причем формула для $T_{1}(6.34)$, в отличие от “тавтологической” формулы (6.31), уже не содержит сингулярностей, т.е. уравнения Бете в ней учтены автоматически.

К сожалению, эта точка зрения пока не привела к решению квантовой задачи, которое могло бы послужить альтернативой анзацу Бете. Дело в том, что в изложенном варианте мы находим одно состояние некоторой квантовой модели, причем параметры этой модели довольно трудно контролировать. Стандартная же постановка задачи иная - для заданной квантовой модели надо найти все состояния.

Пусть квантовая модель задана, т.е. заданы все нули полинома четной степени $\phi(2 x)=\tau^{0,0}(x) \tau^{1,1}(x-1)$. Откуда возникает неоднозначность в определении $\tau^{1,0}(x)$

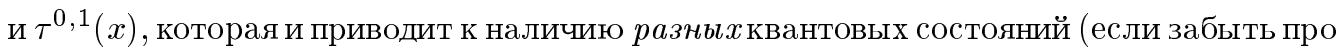
возможное вырождение)? Во-первых, нули $\phi(2 x)$ можно по-разному (но только поровну) распределять меж ду $\tau^{0,0}(x)$ и $\tau^{1,1}(x-1)$. Во-вторых, выбор этого разбиения задает кривую Рейсенарса не однозначно, а с точностью до конечного числа возможностей ${ }^{8}$.

\footnotetext{
7)Это условие означает, что функция $Q(2 x)$ - эллиптический полином, умноженный на экспоненциальную функцию от $x$. Наличие этой экспоненты никак не портит данные выше формулы, где мы считали подобные множители равными единице. В моделях с рациональной или тригонометрической $R$-матрицами такие множители возникают в случае твистованных граничных условий в квантовой модели.

${ }^{8)}$ В системе Калоджеро-Мозера имеется непрерывный аналог этой ситуации. Допустим, что заданы начальные координаты частиц в системе, а начальные импульсы заданы по отношению к одному из высших гамильтоновых потоков. Значения интегралов движения (т.е. спектральная кривая) определяются этими данными с точностью до конечного числа возможностей.
} 
По-видимому, наборы состояний, которые получаются из дискретной свободы первого и второго типов, должны полностью перекрываться.

\section{7. ЗАКЛЮЧЕНИЕ}

Для наглядности сводка изложенных в основном тексте результатов оформлена здесь в виде "словаря" для перевода квантовых понятий на язык теории дискретных солитонных уравнений.

Собственные значения квантовых трансфер-матриц - (классические)

$\tau$-функции. Квантовые трансфер-матрицы коммутируют при всех значениях спектрального параметра и параметров представления во вспомогательном пространстве. Это свойство дает возможность привести их к диагональной форме одновременно. Везде в основной части обзора подразумевалось это диагональное представление.

Правила слияния - уравнения Хироты. Процедура "слияния" во вспомогательном пространстве порождает семейство трансфер-матриц, действующих в одном и том же квантовом пространстве. Они удовлетворяют ряду функциональных соотношений, которые можно привести к модельно-независимой форме. В этой форме соотношения для $T$-матриц $T_{s}^{a}(u)$, отвечаюших прямоугольным диаграммам Юнга, совпадают с 3-членным уравнением Хироты. В общем случае, т.е. для диаграмм Юнга произвольной формы, возникают "высшие" уравнения иерархии билинейных разностных уравнений, которые следуют из соотношений Плюккера (подробности см. в работе [21]).

Фиксация квантовой модели - наложение граничных условий в уравнении Хироты. Тип граничных условий совпадает с таковым для дискретной двумеризованной цепочки Тоды с открытыми концами. Другим важным условием, в конечном счете вытекаюшим из уравнения Янга-Бакстера, является то, что $T_{s}^{a}(u)$ для моделей с эллиптической $R$-матрицей должны быть эллиптическими полиномами от спектрального параметра $u$. Корни этого полинома при $a=0$ - "внешние" данные задачи. Они полностью задают квантовую модель. Эллиптические решения уравнения Хироты с указанными граничными условиями соответствуют разным собственным состояниям гамильтониана квантовой модели.

"Шаг" иерархического анзаца Бете - преобразование Беклунда. Квантовые интегрируемые системы с внутренними степенями свободы решаются применением цепочки (иерархии) последовательных анзацев Бете. Этот метод фактически заключается в интегрировании по некоторым степеням свободы с помощью анзаца типа Бете, причем эффективный гамильтониан опять оказывается интегрируемым. Повторяя эту процедуру несколько раз, можно прийти к модели без внутренних степеней свободы, которая решается обычным анзацем Бете.

Классический образ этой схемы - цепочка преобразований Беклунда для модели Тоды с открытыми концами. Под преобразованием Беклунда мы здесь понимаем переход от решения нелинейного уравнения к (нужным образом нормированному) решению вспомогательных линейных задач. Это последнее удовлетворяет тому же нелинейному 
уравнению, но с более простым граничным условием. Повторяя эту процедуру, можно свести модель Тоды типа $A_{k-1}$ к $A_{1}$-задаче, которая представляет собой дискретное уравнение Лиувилля.

$T$ - $Q$-соотношение Бакстера - одна из форм ВЛЗ для уравнения Хироты. $T$ - $Q$-соотношение Бакстера - основной технический инструмент стандартного подхода - можно рассматривать как разностное уравнение второго порядка на функцию $Q$ c "потенциалом" $T$, где обе эти функции изначально неизвестны. Задание определенных аналитических свойств этих функций позволяет одновременно найти и ту, и другую. В нашей схеме собственные значения $Q$-оператора Бакстера представляют собой подходяшим образом нормированные решения вспомогательных линейных задач для уравнения Хироты. Эта интерпретация в какой-то мере объясняет, почему это знаменитое соотношение, появившееся в работах Бакстера как загадочный технический прием, оказалось столь полезным и универсальным в контексте квантовой интегрируемости. Достаточно вспомнить, что и при решении классических солитонных уравнений решения линейной задачи со специальными аналитическими свойствами (функции Бейкера-Ахиезера) играют ключевую роль.

Система уравнений Бете для иерархического анзаца - уравнения движения моделей типа Калоджеро-Мозера в дискретном времени. Дискретное время $t$ нумерует вершины диаграммы Дынкина. Корни уравнений Бете $u_{j}^{t}$ (в стандартной интерпретации быстроты квазичастиц на уровне $t$ ) интерпретируются как координаты $j$-й частишы в модели типа Калоджеро-Мозера в момент времени $t$. Тем самым уравнения Бете становятся уравнениями движения некоторой динамической системы в дискретном времени.

Зависимость нулей трансфер-матриц $A_{1}$-моделей $T_{s}(u)$ от $s$ - динамика полюсов эллиптических решений дискретного уравнения Лиувилля в “лабораторных" переменных. Любопытно, что одна из первых статей по алгебраическому анзацу Бете [66] и статья [61], в которой исследовалась динамика сингулярностей в решениях уравнения Лиувилля, появились рядом в одном и том же номере журнала “ТМФ" в 1979 г. В то время трудно было предположить, что эти вопросы окажутся настолько тесно связанными между собой.

Мы полагаем, что этот словарь можно продолжить, включив в него не только понятия, относящиеся к спектральным свойствам систем на конечных решетках, но и понятия, нужные для описания динамических и корреляционных свойств. Возможно, для этого надо рассматривать решения того же уравнения Хироты с более сложными аналитическими свойствами. Примеры появления уравнения Хироты в задаче о корреляционных функциях модели Изинга или $X Y$-модели известны уже довольно давно $[4,5]$.

Другое не менее интересное направление, в котором стоит продолжить наш словарь, это возможное отождествление недиагональных элементов квантовых матриц монодромии с какими-то объектами в классических интегрируемых иерархиях. Первый шаг в этом направлении сделан в [67].

Автор благодарен П. Вигману, И. Кричеверу и О. Липану за сотрудничество, а также 
А. Маршакову, А. Миронову, А. Орлову, А. Погребкову, Т. Такебе, С. Харчеву, К. Хасегаве и С. Хорошкину за полезные обсуждения. Работа была частично поддержана грантом РФФИ 97-02-19085 и грантом 96-15-96455 поддержки научных школ.

\section{ПРИЛОЖЕНИЕ}

Грассмановы многообразия и соотношения Плюккера. Билинейные правила слияния для $T$-матриц тесно связаны с соотношениями Плюккера. Их геометрическая интерпретация заключается в описании вложения грассманова многообразия в проективное пространство (см., например, [68]). Связь между билинейными уравнениями Хироты и геометрией грассмановых многообразий хорошо известна [69-71]. Грассмановы многообразия, отвечающие решениям солитонных уравнений общего вида, как правило, бесконечномерны. Однако решения нужного нам типа соответствуют конечномерныцм грассманнианам.

Грассманниан $\mathbf{G}_{N+1}^{M+1}$ - это множество всех $(M+1)$-мерных линейных подпространств в комплексном $(N+1)$-мерном линейном пространстве $\mathbf{C}^{N+1}$. В частности, $\mathbf{G}_{N+1}^{1}-$ комплексное проективное пространство $\mathbf{P}^{N}$. Пусть $X \in \mathbf{G}_{N+1}^{M+1}-$ некоторое такое $(M+1)$-мерное подпространство, натянутое на векторы

$$
\mathbf{x}^{(j)}=\sum_{i=0}^{N} x_{i}^{(j)} \mathbf{e}^{i}, \quad j=1, \ldots, M+1,
$$

где $\mathbf{e}^{i}$ - базисные векторы в $\mathbf{C}^{N+1}$. Объединим множество их координат в прямоугольную $(N+1) \times(M+1)$-матрицу $x_{i}^{(j)}$. Рассмотрим ее миноры размера $(M+1) \times(M+1)$

$$
\operatorname{det}_{p q}\left(x_{i_{p}}^{(q)}\right) \equiv\left(i_{0}, i_{1}, \ldots, i_{M}\right), \quad p, q=0,1, \ldots, M,
$$

полученные выбором $M+1$ строк $i_{0}, i_{1}, \ldots, i_{M}$. Очевидно, что количество этих миноров равно биномиальному коэффишиенту $C_{N+1}^{M+1}$. Они называются плюккеровыми координатами $X$, определены с точностью до общего скалярного множителя и осушествляют плюккерово вложение грассманниана $\mathbf{G}_{N+1}^{M+1}$ в проективное пространство $\mathbf{P}^{d}$, где $d=C_{N+1}^{M+1}-1$.

Образ $\mathbf{G}_{N+1}^{M+1}{ }_{\text {в }} \mathbf{P}^{d}$ представляет собой пересечение квадрик. Координаты $\left(i_{0}, \ldots, i_{M}\right)$ не независимы, а связаны квадратичными соотношениями Плюккера [68]

$$
\left(i_{0}, i_{1}, \ldots, i_{M}\right)\left(j_{0}, j_{1}, \ldots, j_{M}\right)=\sum_{p=0}^{M}\left(j_{p}, i_{1}, \ldots, i_{M}\right)\left(j_{0}, \ldots, j_{p-1}, i_{0}, j_{p+1}, \ldots, j_{M}\right)
$$

для всех $i_{p}, j_{p}, p=0,1, \ldots, M$.

Билинейные детерминантные тождества. Приведем общую схему получения билинейных детерминантных тождеств из соотношений Плюккера. Чтобы уменьшить число слагаемых в правой части соотношения Плюккера, положим $i_{p}=j_{p}$ для $p \neq$

4 Теоретическая и математическая физика, т. 116, № 1, 1998 г. 
$0,1, \ldots, n$. Тогда все члены в сумме в правой части, кроме первых $n+1$, будут равны нулю, поскольку в соответствуюших минорах найдутся совпадаюшие строки. Для этого случая перепишем соотношения Плюккера следуюшим образом:

$$
\left[i_{0}, i_{1}, \ldots, i_{n}\right] \cdot\left[j_{0}, j_{1}, j_{2}, \ldots, j_{n}\right]=\sum_{p=0}^{n}\left[j_{p}, i_{1}, i_{2}, \ldots, i_{n}\right] \cdot\left[j_{0}, \ldots, j_{p-1}, i_{0}, j_{p+1}, \ldots, j_{n}\right],
$$

где введено обозначение

$$
\left[i_{0}, \ldots, i_{n}\right] \equiv\left(i_{0}, \ldots, i_{n-1}, i_{n}, \ldots, i_{M}\right) .
$$

Это обозначение подразумевает, что все аргументы с индексом, большим $n$, одинаковы во всех членах уравнения. Отличаются только первые $n$ аргументов, которые и вьписаны явно. Например, при $n=1$ имеем

$$
\left[i_{0}, i_{1}\right]\left[j_{0}, j_{1}\right]=\left[j_{0}, i_{1}\right]\left[i_{0}, j_{1}\right]+\left[j_{1}, i_{1}\right]\left[j_{0}, i_{0}\right],
$$

что после изменения обозначений совпадает с (5.8). Следующие ниже доказательства однотипны, они заключаются в применении тождества (5.8) к некоторой специально выбранной прямоугольной матрище.

К ДОКАЗАТЕЛЬСТВУ ТЕОРЕМЫ 5.1. Приведем некоторые подробности доказательства теоремы 5.1. Рассмотрим прямоугольную матрицу $N_{i j}$ размера $(t+2) \times t$, $i=1,2, \ldots, t+2, \quad j=1,2, \ldots, t$ :

$$
\begin{aligned}
& N_{i j}=q_{t+1-j}(u-s-a-2 i+4), \quad 1 \leqslant i \leqslant t-a+1, \\
& N_{i j}=\bar{q}_{t+1-j}(u+s-a-2 i+2 t+5), \quad t-a+2 \leqslant i \leqslant t+2 .
\end{aligned}
$$

Тот факт, что (5.7) удовлетворяет уравнению (5.1), следует из тождества (5.8) для миноров этой матрицы при $i_{1}=1, i_{2}=t-a+1, i_{3}=t-a+2, i_{4}=t+2$. Рассмотрим теперь прямоугольную матрицу $N_{i j}^{\prime}$ размера $(t+3) \times(t+1), i=1,2, \ldots, t+3, j=1,2, \ldots, t+1$ :

$$
\begin{gathered}
N_{1 j}^{\prime}=\delta_{1 j}, \\
N_{i j}^{\prime}=q_{t+2-j}(u-s-a-2 i+4), \quad 2 \leqslant i \leqslant t-a+2, \\
N_{i j}^{\prime}=\bar{q}_{t+2-j}(u+s-a-2 i+2 t+7), \quad t-a+3 \leqslant i \leqslant t+3 .
\end{gathered}
$$

Аналогично из (5.8) при $i_{1}=1, i_{2}=2, i_{3}=t-a+2, i_{4}=t-a+3$ следует (5.2), а при $i_{1}=1, i_{2}=t-a+2, i_{3}=t-a+3, i_{4}=t+1$ следует (5.3).

ОКОНЧАНИЕ ДОКАЗАТЕЛЬСТВА ПРЕДЛОЖЕНИЯ 5.1. Рассмотрим матрицу $N_{i j}^{\prime \prime}$ размера $(t+3) \times(t+1)$ :

$$
\begin{gathered}
N_{1 j}^{\prime \prime}=\delta_{1 j}, \\
N_{2 j}^{\prime \prime}=\delta_{t+1, j}, \\
N_{i j}^{\prime \prime}=q_{t+4-i}(u-2 j+2), \quad 3 \leqslant i \leqslant t+3 .
\end{gathered}
$$

Билинейное соотношение (5.20) следует из (5.8) при $i_{1}=1, i_{2}=2, i_{3}=3, i_{4}=4$. 


\section{Список литературы}

[1] Е.К. Склянин, Л. Д. Фаддеев. ДАН СССР. 1978. Т. 243. С. 1430-1433; Е. К. Склянин. Зап. научн. семин. ЛОМИ. 1980. Т. 95. С. 55-128.

[2] Л. А. Тахтаджия, Л. Д. Фаддеев. Гамильтонов подход в теории солитонов. М.: Наука, 1986.

[3] T. T. Wu, B. M. McCoy, C. A. Tracy, E. Barouch. Phys. Rev. B. 1976. V. 13. P. 316-374.

[4] B. McCoy, T. T. Wu. Phys. Rev. Lett. 1980. V. 45. P. 675-678.

[5] J. H. H. Perk. Phys. Lett. A. 1980. V. 79. P. 3-5.

[6] V. Korepin, A. Izergin, A. Its, N. Slavnov. Int. J. Mod. Phys. B. 1990. V. 4. P. 1003-1037.

[7] N. M. Bogoliubov, A. G. Izergin, V. E. Korepin. Quantum inverse scattering method and correlation functions. Cambridge: Cambridge University Press, 1993.

[8] Al. B. Zamolodchikov. Phys. Lett. B. 1991. V. 253. P. 391-394.

[9] A. Klümper, P. Pearce. Physica A. 1992. V. 183. P. 304-350.

[10] A. Kuniba, T. Nakanishi, J. Suzuki. Int. J. Mod. Phys. A. 1994. V. 9. P. 5215-5312.

[11] R. Hirota. J. Phys. Soc. Japan. 1981. V. 50. P. 3785-3791.

[12] М. Сато, М. Джимбо, Т. Мива. Голономные квантовые поля. М.: Мир, 1983.

[13] D. Bernard, A. LeClair. Phys. Lett. B. 1992. V. 288. P. 113-120; Differential equations for sine-Gordon correlation functions at the free fermion point. Preprint CLNS 94/1276, SPhT-94-021, hep-th/9402144.

[14] A. G. Izergin, D. A. Coker, V. E. Korepin. J. Phys. A. 1992. V. 25. P. 4315-4334.

[15] I. G. Korepanov. Vacuum curves, classical integrable systems in discrete space-time and statistical physics. Preprint, hep-th/9312197.

[16] R. M. Kashaev. Lett. Math. Phys. 1996. V. 35. P. 389-397.

[17] I. Krichever, O. Lipan, P. Wiegmann, A. Zabrodin. Commun. Math. Phys. 1997. V. 188. P. 267-304.

[18] A. Zabrodin. Bethe ansatz and classical Hirota equations. In: Proceedings of the Second A. D. Sakharov International Conference. Moscow, Russia, 20-24 May 1996. Eds I. M. Dremin, A.M. Semikhatov. Singapore: World Scientific, 1997. P. 627-632.

[19] P. Wiegmann. Bethe ansatz and classical Hirota equation, cond-mat/9610132.

[20] A. Zabrodin. Int. J. Mod. Phys. B. 1997. V. 11. P. 3125-3158.

[21] O. Lipan, P. Wiegmann, A. Zabrodin. Mod. Phys. Lett. A. 1997. V. 12. P. 1369-1378.

[22] Л. А. Тахтаджия, Л. Д. Фаддеев. УМН. 1979. Т. 34. № 5. С. 13-63.

[23] P.P. Kulish, N. Yu.Reshetikhin, E. K. Sklyanin. Lett. Math. Phys. 1981. V. 5. P. 393-403.

[24] R. Hirota. J. Phys. Soc. Japan. 1977. V. 43. P. 1424-1433.

[25] R. Hirota. J. Phys. Soc. Japan. 1977. V. 43. P. 2074-2078.

[26] R. Hirota. J. Phys. Soc. Japan. 1977. V. 43. P. 2079-2086.

[27] R. Hirota. J. Phys. Soc. Japan. 1978. V. 45. P. 321-332.

[28] R. Hirota. J. Phys. Soc. Japan. 1979. V. 46. P. 312-319.

[29] А. Забродин. ТМФ. 1997. Т. 113. С. 179-230.

[30] И. В. Чередник. Функц. анализ и его прилож. 1986. Т. 20. № 1. С. 87-88; ДАН СССР. 1985. T. 283. C. $1040-1046$.

[31] M. Jimbo, A. Kuniba, T. Miwa, M. Okado. Commun. Math. Phys. 1988. V. 119. P. 691-696.

[32] А. Г. Изергин, В. Е. Корепин. ДАН СССР. 1981. Т. 259. С. 76-79.

[33] K. Hasegawa. J. Phys. A. 1993. V. 26. P. 3211-3228.

[34] V. Bazhanov, N. Reshetikhin. J. Phys. A. 1990. V. 23. P. 1477-1492.

[35] A. Kuniba, Y. Ohta, J. Suzuki. J. Phys. A. 1995. V. 28. P. 6211-6226.

[36] П. П. Кулиш, Н. Ю. Решетихин. Зап. научн. семин. ЛОМИ. 1982. Т. 120. С. 92-121.

[37] Н. Ю. Решетихин. ЖЭТФ. 1983. Т. 84. С. 1190-1201.

[38] A. Belavin. Nucl. Phys. B. 1980. V. 180. P. 189-200.

[39] M. Richey, C. Tracy. J. Stat. Phys. 1986. V. 42. P. 311-348.

[40] B. Hou, Y. Zhou. J. Phys. A. 1990. V. 23. P. 1147-1154. 
[41] И. В. Чередник. ЯФ. 1982. Т. 36. С. 549-557.

[42] E. К. Склянин. Функц. анализ и его прилож. 1982. Т. 16. № 4. С. 27-34.

[43] Y.-H. Quano. Int. J. Mod. Phys. A. 1994. V. 9. P. 2245-2281.

[44] R. Hirota. J. Phys. Soc. Japan. 1987. V. 56. P. 4285-4288.

[45] A. Bilal, J.-L. Gervais. Nucl. Phys. B. 1989. V. 314. P. 646-686.

[46] S. Kharchev, A. Marshakov, A. Mironov, A. Orlov, A. Zabrodin. Nucl. Phys. B. 1991. V. 366. P. 569-601.

[47] H. Airault, H. McKean, J. Moser. Commun. Pure Appl. Math. 1977. V. 30. P. 95-125.

[48] И. М. Кричевер. Функц. анализ и его прилож. 1980. Т. 14. № 4. С. 45-54.

[49] I. M. Krichever, P. B. Wiegmann, A. V. Zabrodin. Elliptic solutions to difference non-linear equations and related many-body problems. Preprint ITEP-TH-13/97, hep-th/9704090; Commun. Math. Phys. (in print).

[50] S. Saito, N. Saitoh. J. Math. Phys. 1987. V. 28. P. 1052-1055.

[51] И. М. Кричевер, А. В. Забродин. УМН. 1995. Т. 50. № 6. С. 3-56.

[52] I. Krichever, O. Babelon, E. Billey, M. Talon. Spin generalization of the Calogero-Moser system and the Matrix KP equation. Preprint LPTHE 94/42.

[53] А. Н. Кириллов. Зап. научн. семин. ЛОМИ. 1984. Т. 134. С. 169-189.

[54] S. N. M. Ruijsenaars, H. Schneider. Ann. Phys. 1986. V. 170. P. 370-405.

[55] F. Nijhof, O. Ragnisco, V. Kuznetsov. Commun. Math. Phys. 1996. V. 176. P. 681-700.

[56] A. Leznov, M. Saveliev. Physica D. 1981. V. 3. P. 62-72.

[57] A.Н. Лезнов, М. В. Савельев. Групповые методы интегрирования нелинейных динамических систем. М.: Наука, 1985.

[58] E. Frenkel, N. Reshetikhin. Commun. Math. Phys. 1996. V. 178. P. 237-264.

[59] A. Kuniba, J. Suzuki. Commun. Math. Phys. 1995. V. 173. P. 225-264.

[60] J.-L. Gervais, A. Neveu. Nucl. Phys. B. 1984. V. 238. P. 125-141.

[61] Г. П. Джсорджадзе, А. К. Погребков, М. К. Поливанов. ТМФ. 1979. Т. 40. С. 221-234.

[62] G. Jorjadze, A. Pogrebkov, M. Polivanov, S. Talalov. J. Phys. A. 1986. V. 19. P. 121-139.

[63] P. Kulish, E. Sklyanin. Quantum spectral transform method. Recent developments. In: Lect. Notes in Phys, 1982. V. 151. P. 61-119.

[64] R. Baxter. Ann. Phys. 1972. V. 70. P. 193-228.

[65] Р. Бәкстер. Точно решаемые модели в статистической механике. М.: Мир, 1985.

[66] Е. К. Склянин, Л. А. Тахтаджян, Л. Д. Фаддеев. ТМФ. 1979. Т. 40. С. 194-220.

[67] A. Zabrodin. Письма в ЖЭТФ. 1997. Т. 66. С. 620-625; Hidden quantum $R$-matrix in discrete time classical Heisenberg magnet. Preprint ITEP-TH-54/97, solv-int/9710015.

[68] Ф. Гриффитс, Дж.Харрис. Принципы алгебраической геометрии. Т. I, II. М.: Мир, 1982.

[69] M. Sato. RIMS Kokyuroku. 1981. V. 439. P. 30-46.

[70] M. Jimbo, T. Miwa. Publ. RIMS Kyoto Univ. 1983. V. 19. P. 943-1001.

[71] G. Segal, G. Wilson. Publ. IHES. 1985. V. 61. P. 5-65. 